\title{
Classical conformal blocks from TBA for the elliptic Calogero-Moser system
}

\author{
Marcin Piạtek \\ Institute of Physics, University of Szczecin, \\ ul. Wielkopolska 15, 70-451 Szczecin, Poland \\ Bogoliubov Laboratory of Theoretical Physics, Joint Institute for Nuclear Research, \\ 141980 Dubna, Russia \\ E-mail: piatek@fermi.fiz.univ.szczecin.pl
}

Abstract: The so-called Poghossian identities connecting the toric and spherical blocks, the AGT relation on the torus and the Nekrasov-Shatashvili formula for the elliptic Calogero-Moser Yang's (eCMY) functional are used to derive certain expressions for the classical 4-point block on the sphere. The main motivation for this line of research is the longstanding open problem of uniformization of the 4-punctured Riemann sphere, where the 4-point classical block plays a crucial role. It is found that the obtained representation for certain 4-point classical blocks implies the relation between the accessory parameter of the Fuchsian uniformization of the 4-punctured sphere and the eCMY functional. Additionally, a relation between the 4-point classical block and the $N_{f}=4, \mathrm{SU}(2)$ twisted superpotential is found and further used to re-derive the instanton sector of the SeibergWitten prepotential of the $N_{f}=4$, SU(2) supersymmetric gauge theory from the classical block.

Keywords: Supersymmetric gauge theory, Field Theories in Lower Dimensions, Solitons Monopoles and Instantons 


\section{Contents}

1 Introduction 1

2 Classical AGT relation on a sphere 4

2.1 Quantum blocks on torus and sphere 4

$\begin{array}{lll}2.2 & \text { Seiberg-Witten prepotential from the classical 4-point block } & 7\end{array}$

3 Four-point classical blocks from TBA for the eCM system 10

4 Uniformization of 4-punctured sphere and the eCM Yang's functional 13

$\begin{array}{llr}5 & \text { Conclusions } & 20\end{array}$

\section{Introduction}

Let $\mathcal{C}_{g, n}$ be the Riemann surface with genus $g$ and a set of points $z_{1}, \ldots, z_{n}$ removed from it. The basic objects of any two-dimensional conformal field theory (2dCFT) living on $\mathcal{C}_{g}[1,2]$ are the $n$-point correlation functions of primary vertex operators defined on $\mathcal{C}_{g, n}$. Given a marking ${ }^{1} \sigma$ of the Riemann surface $\mathcal{C}_{g, n}$ any correlation function can be factorized according to the pattern given by a pants decomposition of $\mathcal{C}_{g, n}$ and written as a sum (or an integral for theories with a continuous spectrum) which includes the terms consisting of holomorphic and anti-holomorphic conformal blocks times the 3-point functions of the model for each pair of pants. The Virasoro conformal block $\mathcal{F}_{c, \alpha}^{(\sigma)}[\beta](z)$ on $\mathcal{C}_{g, n}$, where $\alpha \equiv\left(\alpha_{1}, \ldots, \alpha_{3 g-3+n}\right), \beta \equiv\left(\beta_{1}, \ldots, \beta_{n}\right)$ depends on the cross ratios of the vertex operators locations denoted symbolically by $\mathrm{z}$ and on the $3 g-3+n$ intermediate conformal weights $\Delta_{\alpha_{i}}=\alpha_{i}\left(Q-\alpha_{i}\right)$. Moreover, it depends on the $n$ external conformal weights $\Delta_{\beta_{a}}=\beta_{a}\left(Q-\beta_{a}\right)$ and on the central charge $c$ which can be parameterized as follows $c=1+6 Q^{2}$ with

$$
Q=b+b^{-1}
$$

Conformal blocks are fully determined by the underlying conformal symmetry. These functions possess an interesting, although not yet completely understood analytic structure. In general, they can be expressed only as a formal power series and no closed formula is known for its coefficients. Among the issues concerning conformal blocks which are still not fully understood there is the problem of their semiclassical limit. This is the limit in which all parameters of the conformal blocks tend to infinity in such a way that their ratios are fixed. It is commonly believed that such limit exists and the conformal blocks behave in this limit exponentially with respect to $z$. This last has been however verified explicitly

\footnotetext{
${ }^{1} \mathrm{~A}$ marking of the Riemann surface $\mathcal{C}_{g, n}$ (for definition see [3]) is a pants decomposition of $\mathcal{C}_{g, n}$ together with the corresponding trivalent graph.
} 
only in the case of the conformal block on the 4-punctured sphere. Indeed, the existence of the semiclassical limit of the 4-point Liouville correlation function and the projection of this correlation function onto an appropriate intermediate conformal family imply that the semiclassical limit of the 4-point conformal block [1] with "heavy" weights $\Delta=b^{-2} \delta$, $\Delta_{i}=b^{-2} \delta_{i}$, with $\delta, \delta_{i}=\mathcal{O}(1)$ has the following form:

$$
\mathcal{F}_{c=1+6 Q^{2}, \Delta}\left[\begin{array}{ll}
\Delta_{3} & \Delta_{2} \\
\Delta_{4} & \Delta_{1}
\end{array}\right](x) \stackrel{b \rightarrow 0}{\longrightarrow} \exp \left\{\frac{1}{b^{2}} \mathrm{f}_{\delta}\left[\begin{array}{ll}
\delta_{3} & \delta_{2} \\
\delta_{4} & \delta_{1}
\end{array}\right](x)\right\} .
$$

The function $\mathrm{f}_{\delta}\left[\begin{array}{ll}\delta_{3} & \delta_{2} \\ \delta_{4} & \delta_{1}\end{array}\right](x)$ is called the classical conformal block [4] or with some abuse of terms, the "classical action" [5,6]. The existence of the semiclassical limit (1.2) has been postulated first in $[5,6]$ where it has been pointed out that the classical block is related to a certain monodromy problem of a null vector decoupling equation in a similar way in which the classical Liouville action is related to the Fuchsian uniformization. This relation has been further used to derive the $\Delta \rightarrow \infty$ limit of the 4-point conformal block and its expansion in powers of the so-called elliptic variable.

Recently, a considerable progress in the theory of conformal blocks and their applications has been achieved. This is mainly due to the discovery of the so-called AGT correspondence by Alday, Gaiotto and Tachikawa in 2009 [7]. The AGT correspondence states that the Liouville field theory (LFT) correlators on $\mathcal{C}_{g, n}$ can be identified with the partition functions $Z_{\mathcal{T}_{g, n}}^{(\sigma)}$ of a class $\mathcal{T}_{g, n}$ (cf. [8]) of four-dimensional $\mathcal{N}=2$ supersymmetric SU(2) quiver gauge theories: ${ }^{2}$

$$
\left\langle\prod_{a=1}^{n} \mathrm{~V}_{\beta_{a}}\right\rangle_{\mathcal{C}_{g, n}}^{\mathrm{LFT}}=Z_{\mathcal{T}_{g, n}}^{(\sigma)}
$$

Let us describe the AGT proposal in more details. As well as the correlators of the Liouville theory, also the partition functions $Z_{\mathcal{T}_{g, n}}^{(\sigma)}$ have an integral representation for a fixed $\sigma$. Indeed, $Z_{\mathcal{T}_{g, n}}^{(\sigma)}$ can be written as the integral over the holomorphic and anti-holomorphic Nekrasov partition functions $[9,10]$ :

$$
Z_{\mathcal{T}_{g, n}}^{(\sigma)}=\int[d a] \mathcal{Z}_{\text {Nekrasov }}^{(\sigma)} \overline{\mathcal{Z}}_{\text {Nekrasov }}^{(\sigma)}
$$

where $[d a]$ is an appropriate measure. The Nekrasov partition function ${ }^{3} \mathcal{Z}_{\text {Nekrasov }}\left(\cdot ; \epsilon_{1}, \epsilon_{2}\right)$ appearing in (1.4) is a product of the three factors:

$$
\mathcal{Z}_{\text {Nekrasov }}=\mathcal{Z}_{\text {class }} \mathcal{Z}_{1 \text {-loop }} \mathcal{Z}_{\text {inst }}
$$

\footnotetext{
${ }^{2}$ Notice, that the Liouville theory correlators are modular [11] and crossing symmetric [12-15] and do not depend on the choice of pants decomposition. This strongly suggests the S-duality invariance of the gauge theory partition functions.

${ }^{3}$ The dot in the first slot symbolizes the dependence on the gauge theory parameters, in general these are: (i) the $3 g-3+n$ gluing parameters $\mathbf{q}=\left(q_{i}=\exp 2 \pi \tau_{i}\right)_{i=1, \ldots, 3 g-3+n}$ associated with the pants decomposition, where $\tau_{i}=\theta_{i} / 2 \pi+4 \pi i / g_{i}^{2}$ are complexified gauge couplings; (ii) the $3 g-3+n$ vacuum expectation values (vev's) of the scalar fields in the vector multiplets a $=\left(a_{1}, \ldots, a_{3 g-3+n}\right)$; (iii) the $n$ mass parameters $\mathrm{m}=\left(m_{1}, \ldots, m_{n}\right)$. Finally, $\epsilon_{1}, \epsilon_{2}$ are the so-called complex $\Omega$-background parameters [54-56].
} 
The first two factors $\mathcal{Z}_{\text {class }} \mathcal{Z}_{1 \text {-loop }} \equiv \mathcal{Z}_{\text {pert }}$ describe the contribution coming from perturbative calculations. Supersymmetry implies that there are contributions to $\mathcal{Z}_{\text {pert }}$ only at tree- $\left(\mathcal{Z}_{\text {class }}\right)$ and 1-loop-level $\left(\mathcal{Z}_{1 \text {-loop }}\right) . \mathcal{Z}_{\text {inst }}$ is the instanton contribution. A significant (Liouville independent, "chiral") part of the AGT conjecture is an exact correspondence between the Virasoro conformal blocks $\mathcal{F}_{c, \alpha}^{(\sigma)}[\beta](\mathrm{z})$ on $\mathcal{C}_{g, n}$ and the instanton sectors $\mathcal{Z}_{\text {inst }}$ of the Nekrasov partition functions of the gauge theories $\mathcal{T}_{g, n}$.

The AGT conjecture was actively studied starting from the moment of its discovery. In particular, various checks $[16,17]$ and proofs $[18-20]$ in special cases have been investigated. Moreover, there have been attempts to explain that conjecture from the points of view of $M$-theory [21, 22] and topological string theory [23]. The direct consequences of AGT relations have been studied for instance in [24-29]. Soon the AGT hypothesis has been extended to the $\mathrm{SU}(\mathrm{N})$-gauge theories $/ W_{\mathrm{N}} 2 \mathrm{dCFT}$ correspondence [30-32]. Extensions to asymptotically free theories [33-35] and five-dimensional gauge theory [36-38] have been analyzed too. Additionally, this duality paved the way for new results in the theory of loop and surface operators [39-43] and has appeared in contexts related to matrix models [44-49]. As previously mentioned, the studies of the AGT duality have inspired several progresses inside pure $2 \mathrm{dCFT}$, namely in the theory of conformal blocks [24, 50-52] as well as in Liouville theory [11].

One of the immediate implications of the AGT proposal is the passibility of a derivation of the instanton contributions to the so-called Seiberg-Witten prepotentials [53] from conformal blocks. Let us recall that originally the Nekrasov partition functions have been introduced to calculate the low energy effective gauge theories prepotential $F\left(\cdot ; \epsilon_{1}, \epsilon_{2}\right)$. For $\epsilon_{1}, \epsilon_{2} \rightarrow 0$, the Nekrasov partition functions behave as follows:

$$
\mathcal{Z}_{\text {Nekrasov }}\left(\cdot ; \epsilon_{1}, \epsilon_{2}\right) \longrightarrow \exp \left\{-\frac{1}{\epsilon_{1} \epsilon_{2}} F\left(\cdot ; \epsilon_{1}, \epsilon_{2}\right)\right\}
$$

where $F\left(\cdot ; \epsilon_{1}, \epsilon_{2}\right)$ becomes the Seiberg-Witten prepotential $F(\cdot)$ in the limit $\epsilon_{1}, \epsilon_{2} \rightarrow 0$.

The Nekrasov functions lead also to an interesting application when one of the parameters $\epsilon_{1}, \epsilon_{2}$ is non-zero. Recently, the limit $\epsilon_{2} \rightarrow 0$ (while $\epsilon_{1}$ is kept finite) has been discussed in [57]. There has been observed that in this limit the Nekrasov partition functions behave as

$$
\mathcal{Z}_{\text {Nekrasov }}\left(\cdot ; \epsilon_{1}, \epsilon_{2}\right) \stackrel{\epsilon_{2} \rightarrow 0}{\longrightarrow} \exp \left\{\frac{1}{\epsilon_{2}} \mathcal{W}\left(\cdot ; \epsilon_{1}\right)\right\}
$$

where $\mathcal{W}\left(\cdot ; \epsilon_{1}\right)=\mathcal{W}_{\text {pert }}\left(\cdot ; \epsilon_{1}\right)+\mathcal{W}_{\text {inst }}\left(\cdot ; \epsilon_{1}\right)$ is the so-called effective twisted superpotential of the corresponding two-dimensional gauge theories restricted to the two-dimensional $\Omega$ background. It is not difficult to realize, looking at particular examples of AGT relations (see next section), that the limit $\epsilon_{2} \rightarrow 0$ should correspond to the classical limit $b \rightarrow 0$ of the conformal blocks.

On the other hand, twisted superpotentials play also a prominent role in another correspondence, the so-called Bethe/gauge correspondence [57-59] which maps supersymmetric vacua of the $\mathcal{N}=2$ theories to Bethe states of quantum integrable systems (QIS's). A result of that duality is that twisted superpotentials are identified with Yang's functionals [60] which describe a spectrum of QIS's. For instance, in [57] Nekrasov and Shatashvili have 
found that the twisted superpotential $\mathcal{W}^{\mathcal{N}=2^{*}, \mathrm{U}(\mathrm{N})}\left(q, a, m ; \epsilon_{1}\right)$ serves as Yang's functional for the N-particle elliptic Calogero-Moser (eCM) system and for the periodic Toda chain (pToda). Hance, by combining the AGT duality and the Bethe/gauge correspondence it is possible to link classical blocks to Yang's functionals. ${ }^{4}$

The main aim of the present work is to exploit this link to shed some light on the classical block on the sphere. The motivation for this line of research is the longstanding open problem of the uniformization of the 4-punctured sphere, where the knowledge of the 4-point classical block plays a key role.

The structure of the paper is as follows. In section 2, quantum blocks on the torus and sphere and its so-called elliptic representations are defined. Afterwards, the classical block on the sphere is introduced and the relation between it and the instanton superpotential of the $\mathrm{SU}$ (2) (or $\mathrm{U}(2)$ ), $N_{f}=4$ theory is written down. This last relation is further used to re-derive in a consistent way the instanton contributions to the Seiberg-Witten prepotential of the $\mathrm{SU}(2)$ (or $\mathrm{U}(2)$ ), $N_{f}=4$ theory starting from the classical block.

In section 3, using the so-called Poghossian identities, the AGT relation on the torus and the Nekrasov-Shatashvili results from [57], the expressions for certain families of classical blocks on the sphere are derived. It turns out that the most non-trivial parts of the classical blocks are encoded in the closed formula of the 2-particle eCM Yang's functional [57].

In section 4, the connection between classical Liouville theory, semiclassical limit of quantum DOZZ Liouville theory and the problem of uniformization of the 4-punctured Riemann sphere is recalled. The role of the classical block on the sphere in that context is clarified. Then, the expressions of certain classical blocks derived in section 3 are applied to calculate the accessory parameter of the Fuchsian uniformization of the 4-punctured sphere. The relation between the accessory parameter and the 2-particle eCM Yang's functional is found.

Finally, section 5 contains conclusions and a discussion of the open problems and possible extensions of the present work.

\section{Classical AGT relation on a sphere}

\subsection{Quantum blocks on torus and sphere}

Let $\hat{q}=\mathrm{e}^{2 \pi i \tau}$ be the elliptic variable on the torus with modular parameter $\tau$ and $x$ be the moduli of the 4-punctured sphere. The quantum conformal blocks on the 1-punctured torus and on the 4-punctured sphere are defined as formal power series:

$$
\begin{aligned}
\mathcal{F}_{c, \Delta}^{\tilde{\Delta}}(q) & =q^{\Delta-\frac{c}{24}}\left(1+\sum_{n=1}^{\infty} \mathcal{F}_{c, \Delta}^{\tilde{\Delta}, n} q^{n}\right), \\
\mathcal{F}_{c, \Delta}^{\tilde{\Delta}, n} & =\sum_{n=|I|=|J|}\left\langle\nu_{\Delta, I}, V_{\tilde{\Delta}}(1) \nu_{\Delta, J}\right\rangle\left[G_{c, \Delta}\right]^{I J},
\end{aligned}
$$

\footnotetext{
${ }^{4}$ Recently, such duality has been established in [61] without employing the AGT conjecture.
} 
and

$$
\begin{aligned}
\mathcal{F}_{c, \Delta}\left[\begin{array}{ll}
\Delta_{3} \Delta_{2} \\
\Delta_{4} \Delta_{1}
\end{array}\right](x) & =x^{\Delta-\Delta_{2}-\Delta_{1}}\left(1+\sum_{n=1}^{\infty} \mathcal{F}_{c, \Delta}^{n}\left[\begin{array}{ll}
\Delta_{3} & \Delta_{2} \\
\Delta_{4} & \Delta_{1}
\end{array}\right] x^{n}\right), \\
\mathcal{F}_{c, \Delta}^{n}\left[\begin{array}{l}
\Delta_{3} \Delta_{2} \\
\Delta_{4}
\end{array}\right] & =\sum_{n=|I|=|J|}\left\langle\nu_{\Delta_{4}}, V_{\Delta_{3}}(1) \nu_{\Delta, I}\right\rangle\left[G_{c, \Delta}\right]^{I J}\left\langle\nu_{\Delta, J}, V_{\Delta_{2}}(1) \nu_{\Delta_{1}}\right\rangle
\end{aligned}
$$

respectively. In the above equations $\left[G_{c, \Delta}\right]^{I J}$ is the inverse of the Gram matrix $\left[G_{c, \Delta}\right]_{I J}=$ $\left\langle\nu_{\Delta, I}, \nu_{\Delta, J}\right\rangle$ of the standard symmetric bilinear form in the Verma module $\mathcal{V}_{\Delta}=\bigoplus_{n=0}^{\infty} \mathcal{V}_{\Delta}^{n}$,

$$
\begin{aligned}
\mathcal{V}_{\Delta}^{n}=\operatorname{Span}\left\{\nu_{\Delta, I}^{n}=L_{-I} \nu_{\Delta}=L_{-i_{k}} \ldots L_{-i_{2}} L_{-i_{1}} \nu_{\Delta}:\right. & \\
I=\left(i_{k} \geq \ldots \geq i_{1} \geq 1\right) & \text { an ordered set of positive integers } \\
& \text { of the length } \left.|I| \equiv i_{1}+\ldots+i_{k}=n\right\} .
\end{aligned}
$$

The operator $V_{\Delta}$ in the matrix elements is the normalized primary chiral vertex operator acting between the Verma modules

$$
\left\langle\nu_{\Delta_{i}}, V_{\Delta_{j}}(z) \nu_{\Delta_{k}}\right\rangle=z^{\Delta_{i}-\Delta_{j}-\Delta_{k}} .
$$

In order to calculate the matrix elements in (2.2), (2.4) it is enough to know the covariance properties of the primary chiral vertex operator with respect to the Virasoro algebra:

$$
\left[L_{n}, V_{\Delta}(z)\right]=z^{n}\left(z \frac{d}{d z}+(n+1) \Delta\right) V_{\Delta}(z), \quad n \in \mathbb{Z} .
$$

As the dimension of $\mathcal{V}_{\Delta}^{n}$ grows rapidly with $n$, the calculations of conformal block coefficients by inverting the Gram matrices become very laborious for higher orders. A more efficient method based on recurrence relations for the coefficients can be used [6, 18, $24,52,62]$.

It is convenient to introduce the so-called elliptic conformal blocks. The 1-point elliptic block $\mathcal{H}_{c, \Delta}^{\tilde{\Delta}}(\hat{q})$ on the torus with modular parameter $\tau$ and elliptic variable $\hat{q}=\mathrm{e}^{2 \pi i \tau}$ is defined by $[18,52]$ :

$$
\mathcal{H}_{c, \Delta}^{\tilde{\Delta}}(\hat{q})=\hat{q}^{\frac{c-1}{24}-\Delta} \eta(\hat{q}) \mathcal{F}_{c, \Delta}^{\tilde{\Delta}}(\hat{q})=\sum_{n=0}^{\infty} \hat{q}^{n} \mathcal{H}_{c, \Delta}^{\tilde{\Delta}, n},
$$

where $\eta(\hat{q})=\hat{q}^{\frac{1}{24}} \prod_{n=1}^{\infty}\left(1-\hat{q}^{n}\right)$ is the Dedekind eta function.

The 4-point elliptic block on the sphere $\mathcal{H}_{c, \Delta}\left[\begin{array}{cc}\Delta_{3} & \Delta_{2} \\ \Delta_{4} & \Delta_{1}\end{array}\right](q)$, where

$$
q \equiv q(x)=\mathrm{e}^{-\pi \frac{K(1-x)}{K(x)}}, \quad K(x)=\int_{0}^{1} \frac{d t}{\sqrt{\left(1-t^{2}\right)\left(1-x t^{2}\right)}}
$$

is defined by [6]

$$
\begin{aligned}
& \mathcal{F}_{c, \Delta}\left[\begin{array}{ll}
\Delta_{3} & \Delta_{2} \\
\Delta_{4} & \Delta_{1}
\end{array}\right](x)=x^{\frac{c-1}{24}-\Delta_{1}-\Delta_{2}}(1-x)^{\frac{c-1}{24}-\Delta_{2}-\Delta_{3}} \\
& \times \theta_{3}(q)^{\frac{c-1}{2}-4\left(\Delta_{1}+\Delta_{2}+\Delta_{3}+\Delta_{4}\right)}(16 q)^{\Delta-\frac{c-1}{24}} \mathcal{H}_{c, \Delta}\left[\begin{array}{cc}
\Delta_{3} & \Delta_{2} \\
\Delta_{4} & \Delta_{1}
\end{array}\right](q),
\end{aligned}
$$


where

$$
\mathcal{H}_{c, \Delta}\left[\begin{array}{ll}
\Delta_{3} & \Delta_{2} \\
\Delta_{4} & \Delta_{1}
\end{array}\right](q)=1+\sum_{n=1}^{\infty}(16 q){ }^{n} \mathcal{H}_{c, \Delta}^{n}\left[\begin{array}{cc}
\Delta_{3} & \Delta_{2} \\
\Delta_{4} & \Delta_{1}
\end{array}\right] .
$$

The coefficients in (2.8) are uniquely determined by the recursion relation: ${ }^{5}$

$$
\mathcal{H}_{c, \Delta}^{n}\left[\begin{array}{ll}
\Delta_{3} & \Delta_{2} \\
\Delta_{4} & \Delta_{1}
\end{array}\right]=\sum_{\substack{r \geq 1 s \geq 1 \\
n \geq r s \geq 1}} \frac{R_{c}^{r s}\left[\begin{array}{ll}
\Delta_{3} & \Delta_{2} \\
\Delta_{4} & \Delta_{1}
\end{array}\right]}{\Delta-\Delta_{r s}(c)} \mathcal{H}_{c, \Delta_{r s}(c)+r s}^{n-r s}\left[\begin{array}{cc}
\Delta_{3} & \Delta_{2} \\
\Delta_{4} & \Delta_{1}
\end{array}\right], \quad n>0
$$

The toric and spherical blocks appear in the best known examples of the "chiral" AGT relations.

1. $\mathcal{F}_{c, \Delta}^{\tilde{\Delta}}(\hat{q})$ is identified with the Nekrasov instanton partition function of the $\mathcal{N}=2^{*}$, $\mathrm{SU}(2)$ gauge theory (which equals to $\left[\mathcal{Z}_{\text {inst }}^{\mathrm{U}(1)}\right]^{-1} \times \mathcal{Z}_{\text {inst }}^{\mathrm{U}(2)}$ as it is written in the second line of the equation below):

$$
\begin{aligned}
\hat{q}^{\frac{c}{24}-\Delta} \mathcal{F}_{c, \Delta}^{\tilde{\Delta}}(\hat{q}) & =\mathcal{Z}_{\text {inst }}^{\mathcal{N}=2^{*}, \mathrm{SU}(2)}\left(\hat{q}, a, m ; \epsilon_{1}, \epsilon_{2}\right) \\
& =\hat{\eta}(\hat{q})^{1-2 \tilde{\Delta}} \mathcal{Z}_{\text {inst }}^{\mathcal{N}=2^{*}, \mathrm{U}(2)}\left(\hat{q}, a, m ; \epsilon_{1}, \epsilon_{2}\right) .
\end{aligned}
$$

In eq. (2.10) $\hat{\eta}(\hat{q}) \equiv \prod_{n=1}^{\infty}\left(1-\hat{q}^{n}\right)$ and the torus block parameters, namely the external conformal weight $\tilde{\Delta}$, the intermediate weight $\Delta$ and the Virasoro central charge $c$ can be expressed in terms of the $\mathcal{N}=2^{*}, \mathrm{SU}(2)$ super-Yang-Mills theory parameters as follows

$$
\tilde{\Delta}=-\frac{m\left(m+\epsilon_{1}+\epsilon_{2}\right)}{\epsilon_{1} \epsilon_{2}}, \quad \Delta=\frac{\left(\epsilon_{1}+\epsilon_{2}\right)^{2}-4 a^{2}}{4 \epsilon_{1} \epsilon_{2}}, \quad c=1+6 \frac{\left(\epsilon_{1}+\epsilon_{2}\right)^{2}}{\epsilon_{1} \epsilon_{2}} \equiv 1+6 Q^{2} .
$$

In eq. (2.11) $m$ is the mass of the adjoint hypermultiplet, $a$ is the vacuum expectation value (vev) of the complex scalar of the gauge multiplet and $\epsilon_{1}, \epsilon_{2}$ are $\Omega$-background parameters.

2. $\mathcal{F}_{c, \Delta}\left[\begin{array}{ll}\Delta_{3} & \Delta_{2} \\ \Delta_{4} & \Delta_{1}\end{array}\right](x)$ is related to the Nekrasov instanton partition function of the $\mathcal{N}=2$, $\mathrm{SU}(2)$ (or U(2)) super-Yang-Mills theory with four flavors $\left(N_{f}=4\right)$ :

$$
\begin{aligned}
x^{\Delta_{1}+\Delta_{2}-\Delta} \mathcal{F}_{c, \Delta}\left[\begin{array}{cc}
\Delta_{3} & \Delta_{2} \\
\Delta_{4} & \Delta_{1}
\end{array}\right](x) & =\mathcal{Z}_{\text {inst }}^{N_{f}=4, \mathrm{SU}(2)}\left(x, a, \mu_{i} ; \epsilon_{1}, \epsilon_{2}\right) \\
& =(1-x)^{-\frac{\left(\mu_{1}+\mu_{2}\right)\left(\mu_{3}+\mu_{4}\right)}{2 \epsilon_{1} \epsilon_{2}}} \mathcal{Z}_{\text {inst }}^{N_{f}=4, \mathrm{U}(2)}\left(x, a, \mu_{i} ; \epsilon_{1}, \epsilon_{2}\right) .
\end{aligned}
$$

In eq. (2.12) the central charge $c$, the intermediate weight $\Delta$ and the four external weights $\Delta_{i}$ are related to the vev $a$, the masses $\mu_{1}, \ldots, \mu_{4}$ of the four hypermultiplets

\footnotetext{
${ }^{5}$ Here, for the central charge parameterized as $c=1+6\left(b+\frac{1}{b}\right)^{2} \equiv 1+6 Q^{2}$ the degenerate conformal weights are of the form $\Delta_{r s}(c)=\frac{1-r^{2}}{4} b^{2}+\frac{1-r s}{2}+\frac{1-s^{2}}{4} \frac{1}{b^{2}}$. The explicit form of the coefficients $R_{c}^{r s}\left[\begin{array}{cc}\Delta_{3} & \Delta_{2} \\ \Delta_{4} & \Delta_{1}\end{array}\right]$ is known [63].
} 
and the $\Omega$-background parameters $\epsilon_{1}, \epsilon_{2}$ respectively by the following relations:

$$
\begin{aligned}
c & =1+6 \frac{\left(\epsilon_{1}+\epsilon_{2}\right)^{2}}{\epsilon_{1} \epsilon_{2}} \equiv 1+6 Q^{2}, & \Delta & =\frac{\left(\epsilon_{1}+\epsilon_{2}\right)^{2}-4 a^{2}}{4 \epsilon_{1} \epsilon_{2}}, \\
\Delta_{1} & =\frac{\frac{1}{4}\left(\epsilon_{1}+\epsilon_{2}\right)^{2}-\frac{1}{4}\left(\mu_{1}-\mu_{2}\right)^{2}}{\epsilon_{1} \epsilon_{2}}, & \Delta_{2} & =\frac{\frac{1}{2}\left(\mu_{1}+\mu_{2}\right)\left(\epsilon_{1}+\epsilon_{2}-\frac{1}{2}\left(\mu_{1}+\mu_{2}\right)\right)}{\epsilon_{1} \epsilon_{2}}, \\
\Delta_{3} & =\frac{\frac{1}{2}\left(\mu_{3}+\mu_{4}\right)\left(\epsilon_{1}+\epsilon_{2}-\frac{1}{2}\left(\mu_{3}+\mu_{4}\right)\right)}{\epsilon_{1} \epsilon_{2}}, & \Delta_{4} & =\frac{\frac{1}{4}\left(\epsilon_{1}+\epsilon_{2}\right)^{2}-\frac{1}{4}\left(\mu_{3}-\mu_{4}\right)^{2}}{\epsilon_{1} \epsilon_{2}} .
\end{aligned}
$$

The identities (2.10) and (2.12) are understood as equalities between the coefficients of the expansions of both sides in powers of $\hat{q}$ and $x$ respectively. ${ }^{6}$ In both the above examples the background charge is given by

$$
Q=\sqrt{\frac{\epsilon_{2}}{\epsilon_{1}}}+\sqrt{\frac{\epsilon_{1}}{\epsilon_{2}}} .
$$

In order to be consistent with the standard parametrization (1.1) we shall set:

$$
\sqrt{\frac{\epsilon_{2}}{\epsilon_{1}}}=b
$$

\subsection{Seiberg-Witten prepotential from the classical 4-point block}

The asymptotic behavior (1.2) implies the following expansion of the 4-point classical block:

$$
\begin{aligned}
\mathrm{f}_{\delta}\left[\begin{array}{ll}
\delta_{3} & \delta_{2} \\
\delta_{4} & \delta_{1}
\end{array}\right](x) & =\left(\delta-\delta_{1}-\delta_{2}\right) \log x+\sum_{n=1}^{\infty} x^{n} \mathrm{f}_{\delta}^{n}\left[\begin{array}{ll}
\delta_{3} & \delta_{2} \\
\delta_{4} & \delta_{1}
\end{array}\right] \\
& =\left(\delta-\delta_{1}-\delta_{2}\right) \log x+\frac{\left(\delta+\delta_{3}-\delta_{4}\right)\left(\delta+\delta_{2}-\delta_{1}\right)}{2 \delta} x+\ldots
\end{aligned}
$$

The coefficients $\mathrm{f}_{\delta}^{n}\left[\begin{array}{ll}\delta_{3} & \delta_{2} \\ \delta_{4} & \delta_{1}\end{array}\right]$ in (2.15) are calculated directly from the limit (1.2) and the power expansion of the quantum block:

$$
\begin{aligned}
\sum_{n=1}^{\infty} x^{n} \mathfrak{f}_{\delta}^{n}\left[\begin{array}{ll}
\delta_{3} & \delta_{2} \\
\delta_{4} & \delta_{1}
\end{array}\right] & =\lim _{b \rightarrow 0} b^{2} \log \left(1+\sum_{n=1}^{\infty} \mathcal{F}_{c, \Delta}^{n}\left[\begin{array}{ll}
\Delta_{3} & \Delta_{2} \\
\Delta_{4} & \Delta_{1}
\end{array}\right] x^{n}\right) \\
& =\lim _{b \rightarrow 0} b^{2} \log \left(1+\frac{\left(\Delta+\Delta_{3}-\Delta_{4}\right)\left(\Delta+\Delta_{2}-\Delta_{1}\right)}{2 \Delta} x^{n}+\ldots\right),
\end{aligned}
$$

where on the r.h.s. one first expands the logarithm into power series and then the limit is taken of each term separately.

On the other hand the representation (2.7) and the asymptotic behavior (1.2) imply another representation for the classical 4-point block on the sphere:

$$
\begin{aligned}
\mathrm{f}_{\delta}\left[\begin{array}{ll}
\delta_{3} & \delta_{2} \\
\delta_{4} & \delta_{1}
\end{array}\right](x)= & \left(\frac{1}{4}-\delta_{1}-\delta_{2}\right) \log x+\left(\frac{1}{4}-\delta_{2}-\delta_{3}\right) \log (1-x) \\
& +\left(3-4\left(\delta_{1}+\delta_{2}+\delta_{3}+\delta_{4}\right)\right) \log \left(\theta_{3}(q(x))\right) \\
& +\left(\delta-\frac{1}{4}\right) \log (16 q(x))+\mathrm{h}_{\delta}\left[\begin{array}{l}
\delta_{3} \delta_{2} \\
\delta_{4} \delta_{1}
\end{array}\right](q(x))
\end{aligned}
$$

${ }^{6}$ For definition of the expansion coefficients of $\mathcal{Z}_{\text {inst }}^{\mathcal{N}=2^{*}, \mathrm{U}(2)}$ and $\mathcal{Z}_{\text {inst }}^{N_{f}=4, \mathrm{U}(2)}$ see $[9,57]$. 
where the elliptic classical block $\mathrm{h}_{\delta}\left[\begin{array}{ll}\delta_{3} & \delta_{2} \\ \delta_{4} & \delta_{1}\end{array}\right](q)$ is defined by the expansion

$$
\mathrm{h}_{\delta}\left[\begin{array}{ll}
\delta_{3} & \delta_{2} \\
\delta_{4} & \delta_{1}
\end{array}\right](q)=\sum_{n=1}^{\infty}(16 q)^{n} \mathbf{h}_{\delta}^{n}\left[\begin{array}{ll}
l_{3} & \delta_{2} \\
\delta_{4} & \delta_{1}
\end{array}\right] \text {. }
$$

The coefficients $\mathrm{h}_{\delta}^{n}\left[\begin{array}{ll}\delta_{3} & \delta_{2} \\ \delta_{4} & \delta_{1}\end{array}\right]$ are obtained step by step from the limit (1.2):

$$
\sum_{n=1}^{\infty}(16 q)^{n} \mathrm{~h}_{\delta}^{n}\left[\begin{array}{ll}
\delta_{3} & \delta_{2} \\
\delta_{4} & \delta_{1}
\end{array}\right]=\lim _{b \rightarrow 0} b^{2} \log \left(1+\sum_{n=1}^{\infty}(16 q)^{n} \mathcal{H}_{c, \Delta}^{n}\left[\begin{array}{cc}
\Delta_{3} & \Delta_{2} \\
\Delta_{4} & \Delta_{1}
\end{array}\right]\right)
$$

As mentioned in the Introduction classical blocks correspond to twisted superpotentials. In the case under consideration, joining together (1.2), (1.7), (2.12) and (2.14), one gets

$$
\begin{aligned}
\left(\delta_{1}+\delta_{2}-\delta\right) \log x+\mathrm{f}_{\delta}\left[\begin{array}{cc}
\delta_{3} & \delta_{2} \\
\delta_{4} & \delta_{1}
\end{array}\right](x) & =\frac{1}{\epsilon_{1}} \mathcal{W}_{\text {inst }}^{N_{f}=4, \mathrm{SU}(2)}\left(x, a, \mu_{i} ; \epsilon_{1}\right) \\
& =\frac{1}{\epsilon_{1}} \mathcal{W}_{\text {inst }}^{N_{f}=4, \mathrm{U}(2)}\left(x, a, \mu_{i} ; \epsilon_{1}\right)-\frac{\left(\mu_{1}+\mu_{2}\right)\left(\mu_{3}+\mu_{4}\right)}{2 \epsilon_{1}^{2}} \log (1-x),
\end{aligned}
$$

where the classical weights are defined by $\delta \equiv \lim _{b \rightarrow 0} b^{2} \Delta=\lim _{\epsilon_{2} \rightarrow 0} \frac{\epsilon_{2}}{\epsilon_{1}} \Delta$ and $\delta_{i} \equiv$ $\lim _{b \rightarrow 0} b^{2} \Delta_{i}=\lim _{\epsilon_{2} \rightarrow 0} \frac{\epsilon_{2}}{\epsilon_{1}} \Delta_{i}$. Explicitly, they read as follows

$$
\begin{array}{rlrl}
\delta=\frac{1}{4}-\frac{a^{2}}{\epsilon_{1}^{2}}, & \delta_{1} & =\frac{1}{4}-\frac{\left(\mu_{1}-\mu_{2}\right)^{2}}{4 \epsilon_{1}^{2}}, & \delta_{2}=\frac{\mu_{1}+\mu_{2}}{2 \epsilon_{1}}-\frac{\left(\mu_{1}+\mu_{2}\right)}{4 \epsilon_{1}^{2}} \\
\delta_{3}=\frac{\mu_{3}+\mu_{4}}{2 \epsilon_{1}}-\frac{\left(\mu_{3}+\mu_{4}\right)^{2}}{4 \epsilon_{1}^{2}}, & \delta_{4}=\frac{1}{4}-\frac{\left(\mu_{3}-\mu_{4}\right)^{2}}{4 \epsilon_{1}^{2}} .
\end{array}
$$

The relation (2.19) gives rise to a self-consistent method to calculate from the classical block the instanton prepotential for the $\mathrm{SU}(2)$ (or $\mathrm{U}(2)$ ), $N_{f}=4$ theory (cf. [64]). First, notice that from (1.6) and (1.7) one gets

$$
\mathcal{W}\left(\cdot ; \epsilon_{1}\right) \stackrel{\epsilon_{1} \rightarrow 0}{\longrightarrow}-\frac{F(\cdot)}{\epsilon_{1}} .
$$

Then, using the above formula and eq. (2.19) with the values of the classical weights expressed in (2.20) one can write the following relation in the case of the SU(2) theory:

$$
\begin{aligned}
F_{\text {inst }}^{N_{f}=4, \mathrm{SU}(2)}\left(x, a, \mu_{i}\right) & =-\lim _{\epsilon_{1} \rightarrow 0} \epsilon_{1} \mathcal{W}_{\text {inst }}^{N_{f}=4, \mathrm{SU}(2)}\left(x, a, \mu_{i} ; \epsilon_{1}\right) \\
& =-\lim _{\epsilon_{1} \rightarrow 0}\left(\epsilon_{1}^{2} \mathrm{f}_{\delta}\left[\begin{array}{cc}
\delta_{3} & \delta_{2} \\
\delta_{4} & \delta_{1}
\end{array}\right](x)+\epsilon_{1}^{2}\left(\delta_{1}+\delta_{2}-\delta\right) \log x\right) .
\end{aligned}
$$

Analogously, for the $\mathrm{U}(2)$ theory one gets:

$$
\begin{aligned}
F_{\text {inst }}^{N_{f}=4, \mathrm{U}(2)}\left(x, a, \mu_{i}\right)= & -\lim _{\epsilon_{1} \rightarrow 0} \epsilon_{1} \mathcal{W}_{\text {inst }}^{N_{f}=4, \mathrm{U}(2)}\left(x, a, \mu_{i} ; \epsilon_{1}\right) \\
= & -\lim _{\epsilon_{1} \rightarrow 0}\left(\epsilon_{1}^{2} \mathrm{f}_{\delta}\left[\begin{array}{l}
\delta_{3} \\
\delta_{4} \\
\delta_{1}
\end{array}\right](x)+\epsilon_{1}^{2}\left(\delta_{1}+\delta_{2}-\delta\right) \log x\right) \\
& -\frac{1}{2}\left(\mu_{1}+\mu_{2}\right)\left(\mu_{3}+\mu_{4}\right) \log (1-x) .
\end{aligned}
$$


Eq. (2.22) and (2.23) imply that:

$$
F_{\text {inst }}^{N_{f}=4, \mathrm{U}(2)}\left(x, a, \mu_{i}\right)=F_{\text {inst }}^{N_{f}=4, \mathrm{SU}(2)}\left(x, a, \mu_{i}\right)-\frac{1}{2}\left(\mu_{1}+\mu_{2}\right)\left(\mu_{3}+\mu_{4}\right) \log (1-x) .
$$

The expansion of the instanton SU(2) prepotential can be obtained from eq. (2.22) after using the representation (2.15) of the classical block. The result is the expansion in the bare coupling $x$ :

$$
\begin{aligned}
F_{\text {inst }}^{N_{f}=4, \operatorname{SU}(2)}\left(x, a, \mu_{i}\right)= & \frac{a^{4}+a^{2}\left(\mu_{1} \mu_{2}+\mu_{3} \mu_{4}\right)+\mu_{1} \mu_{2} \mu_{3} \mu_{4}}{2 a^{2}} x \\
& +\left[\frac { 1 } { 6 4 a ^ { 6 } } \left(13 a^{8}+5\left(\mu_{1} \mu_{2} \mu_{3} \mu_{4}\right)^{2}\right.\right. \\
& +a^{6}\left(\mu_{1}^{2}+\mu_{2}^{2}+\mu_{3}^{2}+\mu_{4}^{2}+16 \mu_{1} \mu_{2}+16 \mu_{3} \mu_{4}\right) \\
& +a^{4}\left(16 \mu_{1} \mu_{2} \mu_{3} \mu_{4}+\left(\mu_{3} \mu_{4}\right)^{2}+\left(\mu_{2} \mu_{3}\right)^{2}+\left(\mu_{2} \mu_{4}\right)^{2}+\mu_{1}^{2}\left(\mu_{2}^{2}+\mu_{3}^{2}+\mu_{4}^{2}\right)\right) \\
& \left.\left.-3 a^{2}\left(\left(\mu_{2} \mu_{3} \mu_{4}\right)^{2}+\left(\mu_{1} \mu_{3} \mu_{4}\right)^{2}+\left(\mu_{1} \mu_{2} \mu_{3}\right)^{2}+\left(\mu_{1} \mu_{2} \mu_{4}\right)^{2}\right)\right)\right] x^{2}+\ldots
\end{aligned}
$$

Analogously, an expansion of the instanton SU(2) prepotential with respect to the "renormalized" coupling $q(x)$ may be derived from eq. (2.22) after applying the representation (2.16) of the conformal block:

$$
\begin{aligned}
F_{\text {inst }}^{N_{f}=4, \operatorname{SU}(2)}\left(x, a, \mu_{i}\right)= & a^{2} \log \left(\frac{16 q(x)}{x}\right)-\frac{1}{4}\left(\left(\mu_{1}+\mu_{2}\right)^{2}+\left(\mu_{3}+\mu_{4}\right)^{2}\right) \log (1-x) \\
& -\sum_{i=1}^{4} \mu_{i}^{2} \log \left(\frac{2}{\pi} K(x)\right)-\lim _{\epsilon_{1} \rightarrow 0} \epsilon_{1}^{2} \mathrm{~h}_{\delta}\left[\begin{array}{c}
\delta_{3} \delta_{2} \\
\delta_{4} \delta_{1}
\end{array}\right](q(x)),
\end{aligned}
$$

where for example up to $q^{2}$ :

$$
\begin{aligned}
-\lim _{\epsilon_{1} \rightarrow 0} \epsilon_{1}^{2} \mathbf{h}_{\delta}\left[\begin{array}{cc}
\delta_{3} \delta_{2} \\
\delta_{4} & \delta_{1}
\end{array}\right](q)= & 8 a^{-2} \mu_{1} \mu_{2} \mu_{3} \mu_{4} q \\
& +\left(4 a^{-2}\left[\left(\mu_{3} \mu_{4}\right)^{2}+\left(\mu_{2} \mu_{3}\right)^{2}+\left(\mu_{2} \mu_{4}\right)^{2}+\mu_{1}^{2}\left(\mu_{2}^{2}+\mu_{3}^{2}+\mu_{4}^{2}\right)\right]\right. \\
& -12 a^{-4}\left[\left(\mu_{2} \mu_{3} \mu_{4}\right)^{2}+\mu_{1}^{2}\left(\left(\mu_{3} \mu_{4}\right)^{2}+\mu_{2}^{2}\left(\mu_{3}^{2}+\mu_{4}^{2}\right)\right)\right] \\
& \left.+20 a^{-6}\left(\mu_{1} \mu_{2} \mu_{3} \mu_{4}\right)^{2}\right) q^{2}+\ldots
\end{aligned}
$$

Expansion (2.25) agrees with the formula obtained from the instanton partition function. To show the consistency of our approach, we plug in the expansion (2.25) in eq. (2.24). The result reproduces in the case of equal masses the expansion of the instanton $\mathrm{U}(2)$ prepotential of ref. [49]:

$$
\begin{aligned}
F_{\text {inst }}^{N_{f}=4, \mathrm{U}(2)}\left(x, a, \mu_{i}=\mu\right)= & \frac{a^{4}+6 a^{2} \mu^{2}+\mu^{4}}{2 a^{2}} x \\
& +\frac{13 a^{8}+100 a^{6} \mu^{2}+22 a^{4} \mu^{4}-12 a^{2} \mu^{6}+5 \mu^{8}}{64 a^{6}} x^{2}+\ldots .
\end{aligned}
$$

Let us close this section by remarking that in the intermediate steps of the calculation of eq. (2.26) it appears also the following important formula for the $N_{f}=4, \mathrm{SU}(2)$ twisted 
superpotential:

$$
\begin{aligned}
& \mathcal{W}_{\text {inst }}^{N_{f}=4, \mathrm{SU}(2)}\left(x, a, \mu_{i} ; \epsilon_{1}\right)=-\frac{a^{2}}{\epsilon_{1}} \log \left(\frac{16 q(x)}{x}\right) \\
& +\left[\frac{\epsilon_{1}}{4}+\frac{1}{4 \epsilon_{1}}\left(\left(\mu_{1}+\mu_{2}\right)^{2}+\left(\mu_{3}+\mu_{4}\right)^{2}\right)\right] \log (1-x) \\
& +\left[\frac{\epsilon_{1}}{2}-\sum_{i=1}^{4} \mu_{i}+\frac{1}{\epsilon_{1}} \sum_{i=1}^{4} \mu_{i}^{2}\right] \log \left(\frac{2}{\pi} K(q)\right)+\epsilon_{1} \mathrm{~h}_{\delta}\left[\begin{array}{l}
\delta_{3} \\
\delta_{4} \delta_{1}
\end{array}\right](q(x)) .
\end{aligned}
$$

In [27] the r.h.s. of eq. (2.26) and more precisely its limit for large values of $a$ has been interpreted as the instanton prepotential of the four-dimensional $N_{f}=4, \mathrm{SU}(2)$ theory written in the renormalized coupling $q(x)$. Probably the same interpretation is valid in the case of the two-dimensional theory determined by $\mathcal{W}_{\text {inst }}^{N_{f}=4, \mathrm{SU}(2)}$.

\section{Four-point classical blocks from TBA for the eCM system}

Let us come back to quantum blocks for a while. There exist amazing relationships between conformal blocks on the torus and sphere known as Poghossian identities [24]. In order to spell out these relations quickly and clearly let us modify a little bit the notation and denote by $\mathcal{H}_{c, \Delta_{\lambda}}^{\tilde{\lambda}}(q)$ the torus elliptic block and by $\mathcal{H}_{c, \Delta_{\lambda}}\left[\begin{array}{ll}\lambda_{3} & \lambda_{2} \\ \lambda_{4} & \lambda_{1}\end{array}\right](q)$ the elliptic block on the sphere, where the parameters $\lambda, \tilde{\lambda}, \lambda_{i}$ are related to the conformal weights in the following way:

$$
\Delta \equiv \Delta_{\lambda}=\frac{1}{4}\left(Q^{2}-\lambda^{2}\right), \quad \tilde{\Delta} \equiv \Delta_{\tilde{\lambda}}=\frac{1}{4}\left(Q^{2}-\tilde{\lambda}^{2}\right), \quad \Delta_{i} \equiv \Delta_{\lambda_{i}}=\frac{1}{4}\left(Q^{2}-\lambda_{i}^{2}\right) .
$$

Additionally, the following parametrization for the central charge is assumed: $c=1+$ $6\left(b+\frac{1}{b}\right)^{2} \equiv 1+6 Q^{2}$. In [52] it has been proved that

$$
\begin{aligned}
\mathcal{H}_{c, \Delta_{\lambda}}^{\tilde{\lambda}}(q) & =\mathcal{H}_{c, \Delta_{\lambda}}\left[\begin{array}{cc}
\frac{\tilde{\lambda}}{2} & \frac{\tilde{\lambda}}{2} \\
\frac{b}{2}-\frac{1}{2 b} & \frac{Q}{2}
\end{array}\right](q) \\
& =\mathcal{H}_{c, \Delta_{\lambda}}\left[\begin{array}{cc}
\frac{\tilde{\lambda}}{2}-\frac{b}{2} & \frac{\tilde{\lambda}}{2}+\frac{b}{2} \\
\frac{1}{2 b} & \frac{1}{2 b}
\end{array}\right](q) \\
& =\mathcal{H}_{c, \Delta_{\lambda}}\left[\begin{array}{cc}
\frac{\tilde{\lambda}}{2}-\frac{1}{2 b} & \frac{\tilde{\lambda}}{2}+\frac{1}{2 b} \\
\frac{b}{2} & \frac{b}{2}
\end{array}\right](q),
\end{aligned}
$$

where the $q$ variable is the elliptic nome introduced in (2.6).

Notice, that thanks to the AGT relation (2.10) and eq. (2.5), the elliptic torus block $\mathcal{H}_{c, \Delta_{\lambda}}^{\tilde{\lambda}}(q)$ can be replaced with the $\mathcal{N}=2^{*}, \mathrm{U}(2)$ instanton partition function:

$$
\mathcal{H}_{c, \Delta_{\lambda}}^{\tilde{\lambda}}(q)=\hat{\eta}(q)^{2-2 \Delta_{\tilde{\lambda}}} \mathcal{Z}_{\text {inst }}^{\mathcal{N}=2^{*}, U(2)}\left(q, a, m ; \epsilon_{1}, \epsilon_{2}\right)
$$

where

$$
\lambda=\frac{2 a}{\sqrt{\epsilon_{1} \epsilon_{2}}}, \quad \tilde{\lambda}=2 \sqrt{\frac{m^{2}}{\epsilon_{1} \epsilon_{2}}+\frac{m}{\sqrt{\epsilon_{1} \epsilon_{2}}}\left(\frac{\epsilon_{1}+\epsilon_{2}}{\sqrt{\epsilon_{1} \epsilon_{2}}}\right)+\frac{\left(\epsilon_{1}+\epsilon_{2}\right)^{2}}{4 \epsilon_{1} \epsilon_{2}}} .
$$


Applying eqs. (2.14) and (1.7), one finds that the identities (3.2) and (3.3) give in the limit $b \rightarrow 0\left(\Longleftrightarrow \epsilon_{2} \rightarrow 0\right)$ :

$$
\begin{aligned}
& \frac{1}{\epsilon_{1}} \mathcal{W}_{\text {inst }}^{\mathcal{N}=2^{*}, \mathrm{U}(2)}\left(q, a, m ; \epsilon_{1}\right)-2 \tilde{\delta} \log \hat{\eta}(q)=\mathrm{h}_{\frac{1}{4}-\frac{a^{2}}{\epsilon_{1}^{2}}}\left[\begin{array}{cc}
\frac{1}{4}\left(\frac{3}{4}-\frac{m}{\epsilon_{1}}\left(1+\frac{m}{\epsilon_{1}}\right)\right) & \frac{1}{4}\left(\frac{3}{4}-\frac{m}{\epsilon_{1}}\left(1+\frac{m}{\epsilon_{1}}\right)\right) \\
\frac{3}{16} & \frac{3}{16}
\end{array}\right](q) \\
& =\mathrm{h}_{\frac{1}{4}-\frac{a^{2}}{\epsilon_{1}^{2}}}\left[\begin{array}{cc}
\frac{1}{4}\left(1-\frac{m^{2}}{\epsilon_{1}^{2}}\right) & -\frac{1}{4} \frac{m}{\epsilon_{1}}\left(2+\frac{m}{\epsilon_{1}}\right) \\
\frac{1}{4} & \frac{1}{4}
\end{array}\right](q),
\end{aligned}
$$

where $\mathcal{W}_{\text {inst }}^{\mathcal{N}=2^{*}, \mathrm{U}(2)}\left(q, a, m ; \epsilon_{1}\right)=\lim _{\epsilon_{2} \rightarrow 0} \epsilon_{2} \log \mathcal{Z}_{\text {inst }}^{\mathcal{N}=2^{*}, \mathrm{U}(2)}\left(q, a, m ; \epsilon_{1}, \epsilon_{2}\right)$ and the classical conformal weights $\tilde{\delta}, \delta$ and $\delta_{i}$ (written explicitly in (3.4)) are defined by

$$
\begin{aligned}
\tilde{\delta} & \equiv \lim _{b \rightarrow 0} b^{2} \Delta_{\tilde{\lambda}}=\lim _{\epsilon_{2} \rightarrow 0} \frac{\epsilon_{2}}{\epsilon_{1}} \Delta_{\tilde{\lambda}}=-\frac{m\left(m+\epsilon_{1}\right)}{\epsilon_{1}^{2}}, \\
\delta & \equiv \lim _{b \rightarrow 0} b^{2} \Delta_{\lambda}=\lim _{\epsilon_{2} \rightarrow 0} \frac{\epsilon_{2}}{\epsilon_{1}} \Delta_{\lambda}=\frac{1}{4}-\frac{a^{2}}{\epsilon_{1}^{2}}, \\
\delta_{i} & \equiv \lim _{b \rightarrow 0} b^{2} \Delta_{\lambda_{i}}=\lim _{\epsilon_{2} \rightarrow 0} \frac{\epsilon_{2}}{\epsilon_{1}} \Delta_{\lambda_{i}} .
\end{aligned}
$$

On the other hand, Nekrasov and Shatashvili have found in [57] that the instanton twisted superpotential $\mathcal{W}_{\text {inst }}^{\mathcal{N}=2^{*}, \mathrm{U}(\mathrm{N})}$ computed from the free energy $\log \mathcal{Z}_{\text {inst }}$ of the onedimensional interacting gas of the $\mathcal{N}=2^{*} \mathrm{U}(\mathrm{N})$ instanton particles gives the thermodynamic Bethe ansatz [60] for the N-particle eCM system: ${ }^{7}$

$$
\mathcal{W}_{\text {inst }}^{\mathcal{N}=2^{*}, \mathrm{U}(\mathrm{N})}\left(q, \mathrm{a}, m ; \epsilon_{1}\right)=\oint_{C} d z\left[-\frac{1}{2} \varphi(z) \log \left(1-q \mathcal{Q}(z) \mathrm{e}^{-\varphi(z)}\right)+\operatorname{Li}_{2}\left(q \mathcal{Q}(z) \mathrm{e}^{-\varphi(z)}\right)\right] .
$$

Here $\varphi(x)$ is the solution of the integral equation:

$$
\varphi(x)=\oint_{C} d y \mathcal{G}(x-y) \log \left(1-q \mathcal{Q}(y) \mathrm{e}^{-\varphi(y)}\right) .
$$

The above solution is determined by the functions $\mathcal{Q}, \mathcal{G}$ and the contour $C$. In [57] it has been found that

$$
\begin{array}{ll}
\mathcal{Q}(x)=\frac{P(x-m) P\left(x+m+\epsilon_{1}\right)}{P(x) P\left(x+\epsilon_{1}\right)}, & P(x)=\prod_{i=1}^{\mathrm{N}}\left(x-a_{i}\right), \\
\mathcal{G}(x)=\frac{d}{d x} \log \frac{\left(x+m+\epsilon_{1}\right)(x-m)\left(x-\epsilon_{1}\right)}{\left(x-m-\epsilon_{1}\right)(x+m)\left(x+\epsilon_{1}\right)} &
\end{array}
$$

where $m$ is mass of the adjoint hypermultiplet and $a_{i}, i=1, \ldots, \mathrm{N}$ are the vev's. The contour $C$ on the complex plane comes from infinity, goes around the points $a_{i}+k \epsilon_{1}$, $i=1, \ldots, \mathrm{N}, k=0,1,2, \ldots$ and goes back to infinity. It separates these points and the points $a_{i}+l m+k \epsilon_{1}, l \in \mathbb{Z}, k=-1,-2, \ldots$.

${ }^{7} \mathrm{Li}_{2}(z)$ is a dilogarithm function defined by

$$
\operatorname{Li}_{2}(z)=\sum_{k=1}^{\infty} \frac{z^{k}}{k^{2}}=\int_{z}^{0} \frac{\log (1-t) d t}{t}
$$


Hence, combining (2.16), (3.4) and (3.5) for $\mathrm{N}=2$ one obtains the following expressions for the two families of the 4-point classical blocks on the sphere:

$$
\begin{aligned}
& \mathrm{f}_{\frac{1}{4}-\frac{a^{2}}{\epsilon_{1}^{2}}}\left[\begin{array}{cc}
\frac{1}{4}\left(\frac{3}{4}-\frac{m}{\epsilon_{1}}\left(1+\frac{m}{\epsilon_{1}}\right)\right) & \frac{1}{4}\left(\frac{3}{4}-\frac{m}{\epsilon_{1}}\left(1+\frac{m}{\epsilon_{1}}\right)\right) \\
\frac{3}{16} & \frac{3}{16}
\end{array}\right](x)=\left(-\frac{1}{8}+\frac{1}{4} \frac{m}{\epsilon_{1}}\left(1+\frac{m}{\epsilon_{1}}\right)\right) \log x \\
& +\left(-\frac{1}{8}+\frac{1}{2} \frac{m}{\epsilon_{1}}\left(1+\frac{m}{\epsilon_{1}}\right)\right) \log (1-x) \\
& +2 \frac{m}{\epsilon_{1}}\left(1+\frac{m}{\epsilon_{1}}\right) \log \left(\theta_{3}(q(x)) \hat{\eta}(q(x))\right) \\
& -\frac{a^{2}}{\epsilon_{1}^{2}} \log (16 q(x))+\frac{1}{\epsilon_{1}} \mathcal{W}_{\text {inst }}^{\mathcal{N}=2^{*}, \mathrm{U}(2)}\left(q(x), \mathrm{a}, m ; \epsilon_{1}\right)
\end{aligned}
$$

and

$$
\begin{aligned}
& \mathrm{f}_{\frac{1}{4}-\frac{a^{2}}{\epsilon_{1}^{2}}}\left[\begin{array}{cc}
\frac{1}{4}\left(1-\frac{m^{2}}{\epsilon_{1}^{2}}\right)-\frac{1}{4} \frac{m}{\epsilon_{1}}\left(2+\frac{m}{\epsilon_{1}}\right) \\
\frac{1}{4} & \frac{1}{4}
\end{array}\right](x)=\frac{1}{4} \frac{m}{\epsilon_{1}}\left(2+\frac{m}{\epsilon_{1}}\right) \log x+\frac{1}{2} \frac{m}{\epsilon_{1}}\left(1+\frac{m}{\epsilon_{1}}\right) \log (1-x) \\
& +2 \frac{m}{\epsilon_{1}}\left(1+\frac{m}{\epsilon_{1}}\right) \log \left(\theta_{3}(q(x)) \hat{\eta}(q(x))\right) \\
& -\frac{a^{2}}{\epsilon_{1}^{2}} \log (16 q(x))+\frac{1}{\epsilon_{1}} \mathcal{W}_{\text {inst }}^{\mathcal{N}=2^{*}, \mathrm{U}(2)}\left(q(x), \mathrm{a}, m ; \epsilon_{1}\right),
\end{aligned}
$$

where $\mathrm{a}=\left(a_{1}, a_{2}\right)=(a,-a)$. Let us emphasize, that the elliptic classical blocks, that so far were known only in the form of power series, in eqs. (3.9) and (3.10) are expressed in terms of the eCM Yang's functional.

Notice, that for $m=0$ or $m=-\epsilon_{1}$, the expressions (3.9) and (3.10) give

$$
\begin{aligned}
\mathrm{f}_{\frac{1}{4}-\frac{a^{2}}{\epsilon_{1}^{2}}}\left[\begin{array}{cc}
\frac{3}{16} & \frac{3}{16} \\
\frac{3}{16} & \frac{3}{16}
\end{array}\right](x)=-\frac{1}{8} \log (x(1-x))-\frac{a^{2}}{\epsilon_{1}^{2}} \log (16 q(x)), \\
\mathrm{f}_{\frac{1}{4}-\frac{a^{2}}{\epsilon_{1}^{2}}}\left[\begin{array}{cc}
\frac{1}{4} & 0 \\
\frac{1}{4} & \frac{1}{4}
\end{array}\right](x)=-\frac{a^{2}}{\epsilon_{1}^{2}} \log (16 q(x)) \\
\mathrm{f}_{\frac{1}{4}-\frac{a^{2}}{\epsilon_{1}^{2}}}\left[\begin{array}{ll}
0 & \frac{1}{4} \\
\frac{1}{4} & \frac{1}{4}
\end{array}\right](x)=-\frac{1}{4} \log x-\frac{a^{2}}{\epsilon_{1}^{2}} \log (16 q(x)) .
\end{aligned}
$$

Indeed, if $m \in\left\{0,-\epsilon_{1}\right\}$ then $\mathcal{Q}=1$ and $\mathcal{G}=0$ (cf. (3.7)). As a consequence, $\varphi=0$ and from (3.5) we have $\mathcal{W}_{\text {inst }}^{\mathcal{N}=2^{*}, \mathrm{U}(2)}=0$. This completes our proof.

In order to check the consistency of eqs. (3.11)-(3.13) one can expand the right hand sides making use of the power expansion of the elliptic nome ${ }^{8}$

$$
q(x)=\frac{x}{16}\left(1+\frac{x}{2}+\frac{21 x^{2}}{64}+\frac{31 x^{3}}{128}+\frac{6257 x^{4}}{32768}+\ldots\right) .
$$

\footnotetext{
${ }^{8}$ This expansion can be found from the inverse $x(q)=\theta_{2}^{4}(q) / \theta_{3}^{4}(q)$ of the nome function where the theta functions are of the form:

$$
\begin{aligned}
& \theta_{2}(q)=2 q^{\frac{1}{4}} \sum_{n=0}^{\infty} q^{n(n+1)}=2 q^{\frac{1}{4}} \prod_{n=1}^{\infty}\left(1-q^{2 n}\right)\left(1+q^{2 n}\right)^{2}, \\
& \theta_{3}(q)=1+2 \sum_{n=1}^{\infty} q^{n^{2}}=\prod_{n=1}^{\infty}\left(1-q^{2 n}\right)\left(1+q^{2 n-1}\right)^{2} .
\end{aligned}
$$
}


Using (3.14) one obtains the expansion

$$
\begin{aligned}
-\frac{1}{8} \log (x(1-x))-\frac{a^{2}}{\epsilon_{1}^{2}} \log (16 q(x))= & \left(-\frac{1}{8}-\frac{a^{2}}{\epsilon_{1}^{2}}\right) \log x+ \\
& +\left(\frac{1}{8}-\frac{1}{2} \frac{a^{2}}{\epsilon_{1}^{2}}\right) x+\left(\frac{1}{16}-\frac{13}{64} \frac{a^{2}}{\epsilon_{1}^{2}}\right) x^{2}+\left(\frac{1}{24}-\frac{23}{192} \frac{a^{2}}{\epsilon_{1}^{2}}\right) x^{3} \\
& +\left(\frac{1}{32}-\frac{2701}{32768} \frac{a^{2}}{\epsilon_{1}^{2}}\right) x^{4}+\left(\frac{1}{40}-\frac{31237}{327680} \frac{a^{2}}{\epsilon_{1}^{2}}\right) x^{5}+\ldots,
\end{aligned}
$$

which coincides with the classical block expansion (2.15) with $\delta=\frac{1}{4}-\frac{a^{2}}{\epsilon_{1}^{2}}$ and $\delta_{i}=\frac{3}{16}$. Analogous checks can be made in the case of eqs. (3.12) and (3.13).

\section{Uniformization of 4-punctured sphere and the eCM Yang's functional}

The classical Liouville theory is the theory of the conformal factor $\phi(z, \bar{z})$ of the hyperbolic metric on $\mathcal{C}_{g, n}$. The conformal factor is a solution to the Liouville equation:

$$
\partial_{z} \partial_{\bar{z}} \phi(z, \bar{z})=\frac{\mu}{2} \mathrm{e}^{\phi(z, \bar{z})} .
$$

The metric on $\mathcal{C}_{g, n}$ is determined by the singular behavior of $\phi$ at the punctures $z_{1}, \ldots, z_{n}$. Consider the case of $\mathcal{C}_{g, n}$ being a punctured sphere $\mathcal{C}_{0, n}$ and choose complex coordinates on $\mathcal{C}_{0, n}$ in such a way that $z_{n}=\infty$. The existence and the uniqueness of the solution of the equation (4.1) on the sphere with the elliptic singularities, namely

$$
\begin{aligned}
& \phi(z, \bar{z})= \begin{cases}-2\left(1-\xi_{j}\right) \log \left|z-z_{j}\right|+O(1) & \text { as } z \rightarrow z_{j}, \quad j=1, \ldots, n-1, \\
-2\left(1+\xi_{n}\right) \log |z|+O(1) & \text { as } z \rightarrow \infty\end{cases} \\
& \text { where } \xi_{i} \in \mathbb{R}^{+} \backslash\{0\} \quad \text { for all } i=1, \ldots, n \quad \text { and } \quad \sum_{i=1}^{n} \xi_{i}<n-2 \text {, }
\end{aligned}
$$

were proved by Picard [65, 66] (see also [67] for a modern proof). That solution can be interpreted as the conformal factor of the complete, hyperbolic metric on $\mathcal{C}_{0, n}=$ $\mathbb{C} \backslash\left\{z_{1}, \ldots, z_{n-1}\right\}$ with conical singularities characterized by opening angles $\theta_{j}=2 \pi \xi_{j}$ at punctures $z_{j}$. A solution of the Liouville equation is known to exist also in the case of parabolic singularities which correspond to $\xi_{j} \rightarrow 0$. In that case the asymptotic behavior of the Liouville field is:

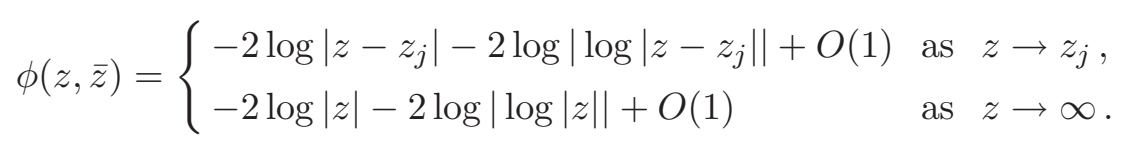

The central statement in classical Liouville theory is the famous uniformization theorem firstly proved by Poincaré and Koebe (1907). The uniformization theorem states that every Riemann surface $\mathcal{C}$ is conformally equivalent:

(i) to the Riemann sphere $\mathbb{C} \cup\{\infty\}$;

(ii) or to the upper half plane $\mathbb{H}=\{\tau \in \mathbb{C}: \mathfrak{I m} \tau>0\}$; 
(iii) or to a quotient of $\mathbb{H}$ by a discrete subgroup $G \subset \operatorname{PSL}(2, \mathbb{R}) \equiv \mathrm{SL}(2, \mathbb{R}) / \mathbb{Z}_{2}$ acting as Möbius transformations.

From this theorem it follows the existence of a meromorphic function

$$
\lambda: \mathbb{H} \ni \tau \rightarrow z=\lambda(\tau) \in \mathbb{H} / G \cong \mathcal{C},
$$

called uniformization. The map $\lambda$ is explicitly known only for the 3-punctured sphere [68] and in a few very special, symmetric cases with higher number of punctures [69]. In particular, an explicit construction of this map for the 4-punctured sphere is a longstanding and still open problem.

One possible method to construct $\lambda$ in the case of the $n$-punctured sphere $\mathcal{C}_{0, n}$ with parabolic singularities has been proposed by Poincaré. This construction is based on the relation of the uniformization problem to a certain Fuchs equation on $\mathcal{C}_{0, n}$. If $\lambda$ is the uniformization of $\mathcal{C}_{0, n}$, the inverse map

$$
\omega=\lambda^{-1}: \mathcal{C}_{0, n} \ni z \rightarrow \tau(z) \in \mathbb{H}
$$

is a multi-valued function with branch points $z_{j}$ and with branches related by the elements $T_{k}$ of the group $G$. One can show that the Schwarzian derivative of $\omega$ is a holomorphic function on $\mathcal{C}_{0, n}$ of the form [69]:

$$
\begin{aligned}
& \{\omega, z\}=\sum_{k=1}^{n-1}\left(\frac{\frac{1}{2}}{\left(z-z_{k}\right)^{2}}+\frac{2 c_{k}}{z-z_{k}}\right), \\
& \{\omega, z\} \stackrel{z \rightarrow \infty}{=} \frac{\frac{1}{2}}{z^{2}}+\mathcal{O}\left(z^{-3}\right),
\end{aligned}
$$

where the so-called accessory parameters $c_{k}$ satisfy the relations

$$
\sum_{k=1}^{n-1} c_{k}=0, \quad \sum_{k=1}^{n-1}\left(4 c_{k} z_{k}+1\right)=1 .
$$

On the other hand it is a well known fact $[69,70]$ that if $\left\{\psi_{1}, \psi_{2}\right\}$ is a fundamental system of normalized $\left(\psi_{1} \psi_{2}^{\prime}-\psi_{1}^{\prime} \psi_{2}=1\right)$ solutions of the Fuchs equation

$$
\psi(z)^{\prime \prime}+\frac{1}{2}\{\omega, z\} \psi(z)=0
$$

with $\mathrm{SL}(2, \mathbb{R})$ monodromy with respect to all punctures then up to a Möbius transformation the inverse map is

$$
\omega=\frac{\psi_{1}}{\psi_{2}}
$$

Therefore, it is possible to reformulate the uniformization problem of $\mathcal{C}_{0, n}$ as a kind of Riemann-Hilbert problem for the Fuchs equation (4.8), where the elements $T_{k} \in G$ correspond to monodromy matrices $M_{k}$ around each puncture $z_{k}$. An applicability of the Fuchs equation to the calculation of the inverse map $\omega$ depends on our ability to calculate the accessory parameters and to choose an appropriate fundamental system of normalized 
solutions. In the case of three punctures the accessory parameters are determined by the equations (4.7). In the case in which $n>3$ the Liouville theory on $\mathcal{C}_{0, n}$ becomes helpful, because eqs. (4.7) do not provide enough constraints in order to calculate the $c_{k}$ 's.

The connection with LFT comes out from the existence of the Poincaré-Klein metric $d \mathrm{~s}_{\mathbb{H}}^{2}=d \tau d \bar{\tau} /(\mathfrak{I m} \tau)^{2}$ on the upper half plane $\mathbb{H}$. The pull back:

$$
\omega^{*} d \mathrm{~s}_{\mathbb{H}}^{2}=\frac{1}{(\mathfrak{I m} \tau)^{2}}\left|\frac{\partial \tau}{\partial z}\right|^{2} d z d \bar{z}=\mathrm{e}^{\phi(z, \bar{z})} d z d \bar{z}
$$

is a regular hyperbolic metric on $\mathcal{C}_{0, n}$, conformal to the standard flat matric $d z d \bar{z}$ on $\mathbb{C}$. The conformal factor $\phi(z, \bar{z})$ satisfies the Liouville equation on $\mathcal{C}_{0, n}$ with the asymptotic condition (4.4). One can show that the energy-momentum tensor $T(z)$ of this solution is equal to one half of the Schwarzian derivative of the inverse map:

$$
T(z) \equiv-\frac{1}{4}\left(\partial_{z} \phi\right)^{2}+\frac{1}{2} \partial_{z}^{2} \phi=\frac{1}{2}\{\omega, z\} .
$$

This allows to calculate all accessory parameters once the classical solution $\phi$ is known. However, the problem of finding solutions of the Liouville equation seems to be at least as hard as the problem of constructing the map $\omega$. For this reason, the application of Liouville theory at this level does not lead to an essential simplification and is not much helpful.

So far we discussed the uniformization problem of the $n$-punctured sphere with parabolic singularities. However, one can consider a more general problem of the sphere with $n$ elliptic singularities characterized by real parameters $\xi_{j}>0$. The notion of accessory parameters can be introduced in terms of the energy-momentum tensor of the solution $\phi(z, \bar{z})$ with the asymptotic condition (4.2). In the present case the energy-momentum tensor is of the form [70]:

$$
\begin{aligned}
& T(z)=\sum_{k=1}^{n-1}\left(\frac{\delta_{k}}{\left(z-z_{k}\right)^{2}}+\frac{c_{k}}{z-z_{k}}\right), \\
& T(z) \stackrel{z \rightarrow \infty}{=} \frac{\delta_{n}}{z^{2}}+\mathcal{O}\left(z^{-3}\right) .
\end{aligned}
$$

The accessory parameters $c_{k}$ obey

$$
\sum_{k=1}^{n-1} c_{k}=0, \quad \sum_{k=1}^{n-1}\left(\delta_{k}+c_{k} z_{k}\right)=\delta_{n},
$$

and as before they are not fully determined for $n>3$. In eqs. (4.10), (4.11) and (4.12) the parameters $\delta_{j}=\frac{1}{4}\left(1-\xi_{j}^{2}\right), j=1, \ldots, n$ are the classical conformal weights. The multivalued function $\omega$ is still of interest. Having $T(z)$ given by (4.10) one can consider the Fuchs equation

$$
\psi(z)^{\prime \prime}+T(z) \psi(z)=0
$$

which monodromy group is a subgroup of $\operatorname{PSL}(2, \mathbb{R})$. The classical result (4.9) holds, where $\omega=\psi_{1} / \psi_{2}$ is the ratio of two linearly independent solutions $\psi_{1}, \psi_{2}$ of the eq. (4.13). Then, 
the map $\omega$ can be computed if one can calculate the accessory parameters in the eq. (4.13) and select fundamental solutions with a suitable monodromy.

For almost a century the problem of accessory parameters has been unsolved until the appearance of the solution proposed by Polyakov (as reported in refs. [70-72]). The so-called Polyakov conjecture states that the properly defined and normalized Liouville action functional evaluated on the classical solution $\phi(z, \bar{z})$ is the generating functional for the accessory parameters:

$$
c_{j}=-\frac{\partial S_{\mathrm{L}}^{\mathrm{cl}}[\phi]}{\partial z_{j}} .
$$

This formula was derived within the path integral approach to the quantum Liouville theory by analyzing the quasi-classical limit of the conformal Ward identity [71]. In the case of parabolic singularities on the $n$-punctured Riemann sphere a rigorous proof has been given by Zograf and Takhtajan [72]. Alternative proofs, valid both in the case of parabolic and general elliptic singularities, have been proposed in $[70,73]$. The Polyakov conjecture can be proved also in the case of the so-called hyperbolic singularities representing "holes" with geodesic boundaries [74].

The next essential step paving the way for an explicit uniformization of the 4-punctured sphere was done by brothers Zamolodchikov [4]. Studying the classical limit of the 4-point function of the quantum Liouville theory they argued that the classical Liouville action on a sphere with for four elliptic (parabolic) singularities can be expressed in terms of the classical Liouville action for three singularities and the 4-point classical block.

Indeed, the 4-point function of the Liouville primary operators located at $\infty, 1, x, 0$ is expressed as an integral over the continuous spectrum of the theory of $s$-channel conformal blocks and DOZZ 3-point functions:

$$
\begin{aligned}
& \left\langle\mathrm{V}_{\alpha_{4}}(\infty, \infty) \mathrm{V}_{\alpha_{3}}(1,1) \mathrm{V}_{\alpha_{2}}(x, \bar{x}) \mathrm{V}_{\alpha_{1}}(0,0)\right\rangle= \\
& \qquad \int_{\frac{Q}{2}+i \mathbb{R}^{+}} d \alpha C\left(\alpha_{4}, \alpha_{3}, \alpha\right) C\left(Q-\alpha, \alpha_{2}, \alpha_{1}\right)\left|\mathcal{F}_{1+6 Q^{2}, \Delta}\left[\begin{array}{c}
\Delta_{3} \Delta_{2} \\
\Delta_{4} \Delta_{1}
\end{array}\right](x)\right|^{2} .
\end{aligned}
$$

Let

$$
\mathbf{1}_{\Delta, \Delta}=\sum_{I}\left(\left|\nu_{\Delta, I}\right\rangle \otimes\left|\nu_{\Delta, I}\right\rangle\right)\left(\left\langle\nu_{\Delta, I}\right| \otimes\left\langle\nu_{\Delta, I}\right|\right)
$$

be the operator that projects onto the space spanned by the states belonging to the conformal family with the highest weight $\Delta$. The correlation function with the $\mathbf{1}_{\Delta, \Delta}$ insertion factorizes into a product of holomorphic and anti-holomorphic factors:

$$
\begin{aligned}
& \left\langle\mathrm{V}_{\alpha_{4}}(\infty, \infty) \mathrm{V}_{\alpha_{3}}(1,1) \mathbf{1}_{\Delta, \Delta} \mathrm{V}_{\alpha_{2}}(x, \bar{x}) \mathrm{V}_{\alpha_{1}}(0,0)\right\rangle= \\
& C\left(\alpha_{4}, \alpha_{3}, \alpha\right) C\left(Q-\alpha, \alpha_{2}, \alpha_{1}\right) \mathcal{F}_{1+6 Q^{2}, \Delta}\left[\begin{array}{cc}
\Delta_{3} & \Delta_{2} \\
\Delta_{4} & \Delta_{1}
\end{array}\right](x) \mathcal{F}_{1+6 Q^{2}, \Delta}\left[\begin{array}{cc}
\Delta_{3} & \Delta_{2} \\
\Delta_{4} & \Delta_{1}
\end{array}\right](\bar{x}) .
\end{aligned}
$$

Assuming a path integral representation for the 1.h.s. and heavy conformal weights $\Delta, \Delta_{i} \sim$ $\frac{1}{b^{2}}$ one should expect in the limit $b \rightarrow 0$ the following asymptotic behavior

$$
\left\langle\mathrm{V}_{\alpha_{4}}(\infty, \infty) \mathrm{V}_{\alpha_{3}}(1,1) \mathbf{1}_{\Delta, \Delta} \mathrm{V}_{\alpha_{2}}(x, \bar{x}) \mathrm{V}_{\alpha_{1}}(0,0)\right\rangle \sim \mathrm{e}^{-\frac{1}{b^{2}} S_{\mathrm{L}}^{\mathrm{cl}}\left(\delta_{i}, x ; \delta\right)}
$$


$S_{\mathrm{L}}^{\text {cl }}\left(\delta_{i}, x ; \delta\right)$ is the 4-point classical Liouville action, i.e. the properly defined Liouville action functional evaluated on the classical solution of the Liouville equation (4.1) on $\mathcal{C}_{0,4}$ with the asymptotic conditions (4.4) or (4.2). The parameters $\delta, \delta_{i}, i=1, \ldots, 4$ are the classical conformal weights defined by $\Delta=\frac{1}{b^{2}} \delta$ and $\Delta_{i}=\frac{1}{b^{2}} \delta_{i}$, where $\delta, \delta_{i}=\mathcal{O}(1)$. On the other hand, one can calculate this limit for the DOZZ coupling constants obtaining $[4,75]$

$$
C\left(\alpha_{4}, \alpha_{3}, \alpha\right) C\left(Q-\alpha, \alpha_{2}, \alpha_{1}\right) \sim \mathrm{e}^{-\frac{1}{b^{2}}\left(S_{\mathrm{L}}^{\mathrm{cl}}\left(\delta_{4}, \delta_{3}, \delta\right)+S_{\mathrm{L}}^{\mathrm{cl}}\left(\delta, \delta_{2}, \delta_{1}\right)\right)} .
$$

$S_{\mathrm{L}}^{\mathrm{cl}}\left(\delta_{1}, \delta_{2}, \delta\right)$ is the 3 -point classical Liouville action which in the case of two parabolic or elliptic weights given respectively by $\delta_{1}=\delta_{2}=\frac{1}{4}$ and $\delta_{i}=\frac{1}{4}\left(1-\xi_{i}^{2}\right), i=1,2$ and one hyperbolic weight $\delta=\frac{1}{4}+p^{2}$ with $p \in \mathbb{R}$ reads as follows $[4,75]$ :

$$
\begin{aligned}
S_{\mathrm{L}}^{\mathrm{cl}}\left(\delta_{1}, \delta_{2}, \delta\right)= & \frac{1}{2}\left(1-\xi_{1}-\xi_{2}\right) \log \mu+\sum_{\sigma_{2}, \sigma_{3}= \pm} F\left(\frac{1-\xi_{1}}{2}+\sigma_{2} \frac{\xi_{2}}{2}+i \sigma_{3} p\right) \\
& -\sum_{j=1}^{2} F\left(\xi_{j}\right)+H(2 i p)+\pi|p|+\text { const },
\end{aligned}
$$

where

$$
F(x)=\int_{1 / 2}^{x} d y \log \frac{\Gamma(y)}{\Gamma(1-y)}, \quad H(x)=\int_{0}^{x} d y \log \frac{\Gamma(-y)}{\Gamma(y)} .
$$

It follows that for $b \rightarrow 0$ the conformal block should have the exponential behavior (1.2). Hence

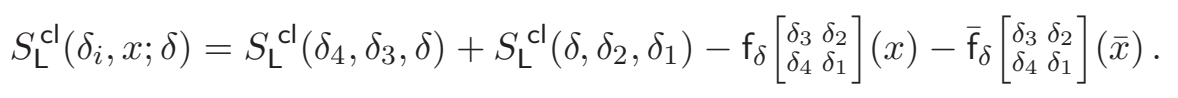

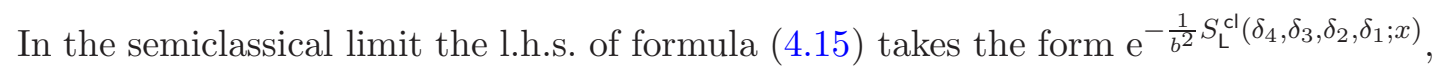
where

$$
S_{\mathrm{L}}^{\mathrm{cl}}\left(\delta_{4}, \delta_{3}, \delta_{2}, \delta_{1} ; x\right) \equiv S_{\mathrm{L}}^{\mathrm{cl}}\left(\delta_{4}, \delta_{3}, \delta_{2}, \delta_{1} ; \infty, 1, x, 0\right) .
$$

The r.h.s. of (4.15) is in this limit determined by the saddle point approximation

$$
\mathrm{e}^{-\frac{1}{b^{2}} S_{\mathrm{L}}^{\mathrm{cl}}\left(\delta_{i}, x\right)} \approx \int_{0}^{\infty} d p \mathrm{e}^{-\frac{1}{b^{2}} S_{\mathrm{L}}^{\mathrm{cl}}\left(\delta_{i}, x ; \delta\right)}
$$

where $\delta=\frac{1}{4}+p_{s}(x)^{2}$ and the saddle point Liouville momentum $p_{s}(x)$ is determined by

$$
\frac{\partial}{\partial p} S_{\mathrm{L}}^{\mathrm{cl}}\left(\delta_{i}, x ; \frac{1}{4}+p^{2}\right)_{\mid p=p_{s}}=0
$$

One thus gets the factorization

$$
\begin{aligned}
S_{\mathrm{L}}^{\mathrm{cl}}\left(\delta_{4}, \delta_{3}, \delta_{2}, \delta_{1} ; x\right)= & S_{\mathrm{L}}^{\mathrm{cl}}\left(\delta_{4}, \delta_{3}, \delta_{s}(x)\right)+S_{\mathrm{L}}^{\mathrm{cl}}\left(\delta_{s}(x), \delta_{2}, \delta_{1}\right) \\
& -\mathrm{f}_{\delta_{s}(x)}\left[\begin{array}{l}
\delta_{3} \delta_{2} \\
\delta_{4} \delta_{1}
\end{array}\right](x)-\overline{\mathrm{f}}_{\delta_{s}(x)}\left[\begin{array}{l}
\delta_{3} \delta_{2} \\
\delta_{4}
\end{array} \delta_{1}\right](\bar{x})
\end{aligned}
$$


first obtained in [4] and as its consistency condition ${ }^{9}$ the classical bootstrap equations:

$$
\begin{aligned}
& S_{\mathrm{L}}^{\mathrm{cl}}\left(\delta_{4}, \delta_{3}, \delta_{s}(x)\right)+S_{\mathrm{L}}^{\mathrm{cl}}\left(\delta_{s}(z), \delta_{2}, \delta_{1}\right)-\mathrm{f}_{\delta_{s}(x)}\left[\begin{array}{ll}
\delta_{3} & \delta_{2} \\
\delta_{4} & \delta_{1}
\end{array}\right](x)-\overline{\mathrm{f}}_{\delta_{s}(x)}\left[\begin{array}{ll}
\delta_{3} & \delta_{2} \\
\delta_{4} & \delta_{1}
\end{array}\right](\bar{x}) \\
& =S_{\mathrm{L}}^{\mathrm{cl}}\left(\delta_{4}, \delta_{1}, \delta_{t}(x)\right)+S_{\mathrm{L}}^{\mathrm{cl}}\left(\delta_{t}(x), \delta_{2}, \delta_{3}\right) \\
& -\mathrm{f}_{\delta_{t}(x)}\left[\begin{array}{ll}
\delta_{1} & \delta_{2} \\
\delta_{4} & \delta_{3}
\end{array}\right](1-x)-\overline{\mathrm{f}}_{\delta_{t}(x)}\left[\begin{array}{ll}
\delta_{1} & \delta_{2} \\
\delta_{4} & \delta_{3}
\end{array}\right](1-\bar{x}) \\
& =2 \delta_{2} \log x \bar{x}+S_{\mathrm{L}}^{\mathrm{cl}}\left(\delta_{1}, \delta_{3}, \delta_{u}(x)\right)+S_{\mathrm{L}}^{\mathrm{cl}}\left(\delta_{u}(x), \delta_{2}, \delta_{4}\right) \\
& -\mathrm{f}_{\delta_{u}(x)}\left[\begin{array}{ll}
\delta_{3} & \delta_{2} \\
\delta_{1} & \delta_{4}
\end{array}\right]\left(\frac{1}{x}\right)-\overline{\mathrm{f}}_{\delta_{u}(x)}\left[\begin{array}{ll}
\delta_{3} & \delta_{2} \\
\delta_{1} & \delta_{4}
\end{array}\right]\left(\frac{1}{\bar{x}}\right), \\
& \delta_{t}(x)=\delta_{s}(1-x), \quad \delta_{u}(x)=\delta_{s}\left(\frac{1}{x}\right) .
\end{aligned}
$$

Turning to the problem of uniformization of the 4-punctured sphere one sees, that having (4.20) one can apply the Polyakov conjecture (4.14) and calculate the accessory parameter of the appropriate Fuchsian equation (cf. [78]). Surprisingly, the twisted superpotential/Yang's functional appears in that context.

According to (2.16) and (3.4) we have

$$
\begin{aligned}
\mathrm{f}_{\delta}\left[\begin{array}{ll}
\delta_{3} & \delta_{2} \\
\delta_{4} & \delta_{1}
\end{array}\right](x)= & \left(\frac{1}{4}-\delta_{1}-\delta_{2}\right) \log x+\left(\frac{1}{4}-\delta_{2}-\delta_{3}\right) \log (1-x) \\
& +\left(3-4\left(\delta_{1}+\delta_{2}+\delta_{3}+\delta_{4}\right)\right) \log \theta_{3}(q(x)) \\
& +\left(\delta-\frac{1}{4}\right) \log (16 q(x))+2 \frac{m\left(m+\epsilon_{1}\right)}{\epsilon_{1}^{2}} \log \hat{\eta}(q(x)) \\
& +\frac{1}{\epsilon_{1}} \mathcal{W}_{\text {inst }}^{\mathcal{N}=2^{*}, \mathrm{U}(2)}\left(q(x), a, m ; \epsilon_{1}\right),
\end{aligned}
$$

where $\delta=\frac{1}{4}-\frac{a^{2}}{\epsilon_{1}^{2}}$ and

$$
\delta_{4}=\delta_{1}=\frac{3}{16}=\frac{1}{4}\left(1-\left(\frac{1}{2}\right)^{2}\right), \quad \delta_{3}=\delta_{2}=\frac{1}{4}\left(1-\xi^{2}\right), \quad \xi=\sqrt{\frac{1}{4}+\frac{m}{\epsilon_{1}}\left(1+\frac{m}{\epsilon_{1}}\right)},
$$

or

$$
\delta_{4}=\delta_{1}=\frac{1}{4}, \quad \delta_{3}=\frac{1}{4}\left(1-\frac{m^{2}}{\epsilon_{1}^{2}}\right), \quad \delta_{2}=-\frac{1}{4} \frac{m}{\epsilon_{1}}\left(2+\frac{m}{\epsilon_{1}}\right) .
$$

Consider the classical block (4.24) with the weights given by (4.25). It can be used to construct the 4-point classical Liouville action:

$$
S_{\mathrm{L}}^{\mathrm{cl}}\left(\delta_{i}, x\right)=S_{\mathrm{L}}^{\mathrm{cl}}\left(\frac{3}{16}, \frac{1}{4}\left(1-\xi^{2}\right), \frac{1}{4}\left(1-\xi^{2}\right), \frac{3}{16}, x ; \frac{1}{4}+p_{s}(x)^{2}\right), \quad 0<\xi<\frac{1}{2},
$$

corresponding to the classical solution on $\mathcal{C}_{0,4}$ with four elliptic singularities characterized by $\xi_{4}=\xi_{1}=\frac{1}{2}$ and $\xi_{3}=\xi_{2}=\xi$. The condition $0<\xi<\frac{1}{2}$, which is fulfilled for $\frac{m}{\epsilon_{1}} \in(-1,0) \backslash\left\{-\frac{1}{2}\right\}$, ensures the existence of such solution ${ }^{10}$ (cf. (4.3)). The saddle point

\footnotetext{
${ }^{9}$ The classical bootstrap equations have been numerically verified for punctures [76] and for punctures and up to two elliptic singularities [77].

${ }^{10}$ Unfortunately, the configuration of the four elliptic singularities with $\xi_{4}=\xi_{3}=\xi_{2}=\xi_{1}=\frac{1}{2}$ for which we have found the classical block (3.11) does not fulfil Picard's inequality (4.3).
} 
momentum $p_{s}(x)$ is determined by the equation (4.21), where $p=i a / \epsilon_{1}$. The Polyakov conjecture in the case under consideration reads

$$
\begin{aligned}
& c_{2}(x)=-\frac{\partial}{\partial x} S_{\mathrm{L}}^{\mathrm{cl}}\left(\delta_{i}, x\right) \\
& =-\left.\frac{\partial}{\partial p} S_{\mathrm{L}}^{\mathrm{cl}}\left(\delta_{i}, x, \frac{1}{4}+p^{2}\right)\right|_{p=p_{s}(x)} \frac{\partial p_{s}(x)}{\partial x}-\left.\frac{\partial}{\partial x} S_{\mathrm{L}}^{\mathrm{cl}}\left(\delta_{i}, x, \frac{1}{4}+p^{2}\right)\right|_{p=p_{s}(x)} \\
& =-\left.\frac{\partial}{\partial x} S_{\mathrm{L}}^{\mathrm{cl}}\left(\delta_{i}, x, \frac{1}{4}+p^{2}\right)\right|_{p=p_{s}(x)}=\left.\frac{\partial}{\partial x} \mathrm{f}_{\frac{1}{4}+p^{2}}\left[\begin{array}{cc}
\frac{1}{4}\left(1-\xi^{2}\right) & \frac{1}{4}\left(1-\xi^{2}\right) \\
\frac{3}{16} & \frac{3}{16}
\end{array}\right](x)\right|_{p=p_{s}(x)} \\
& =\frac{4 \pi K(1-x) p_{s}(x)^{2}-4 \pi E(1-x) p_{s}(x)^{2}-4 \xi^{2} E(x)+E(x)}{8(x-1) x K(x)} \\
& -\frac{\pi E(x) K(1-x) p_{s}(x)^{2}}{2(x-1) x K(x)^{2}}-\frac{4 x-1-4 \xi^{2}}{16(x-1) x} \\
& +\frac{2 m\left(m+\epsilon_{1}\right)}{\epsilon_{1}^{2}} \frac{\partial}{\partial x} \log \hat{\eta}(q(x))+\left.\frac{1}{\epsilon_{1}} \frac{\partial}{\partial x} \mathcal{W}_{\mathrm{inst}}^{\mathcal{N}=2^{*}, \mathrm{U}(2)}\left(q(x), a, m ; \epsilon_{1}\right)\right|_{\frac{i a}{\epsilon_{1}}=p_{s}(x)},
\end{aligned}
$$

where (4.20), (4.19) and (4.24), (4.25) have been used. The symbol $E(x)$ above denotes the complete elliptic integral of the second kind. This calculation shows an amazing relation between the accessory parameter and the instanton part of the $\mathcal{N}=2^{*}, U(2)$ twisted superpotential/Yang's functional. This calculation reveals also the relation between the vev $a$ and the so-called geodesic length functions (cf. [40]):

$$
a=-i \epsilon_{1} p_{s}(x)=-i \frac{\epsilon_{1} \sqrt{\mu}}{4 \pi} \ell_{s}(x) .
$$

Indeed, let us recall that the classical solution $\phi(z, \bar{z})$ on $\mathcal{C}_{0, n}$ describes a unique hyperbolic geometry with singularities at the locations of conformal weights. For elliptic, parabolic and hyperbolic weights one gets conical singularities, punctures and holes with geodesic boundaries, respectively $[75,79]$. In the latter case the (classical) conformal weight $\delta$ is related to the length $\ell$ of the corresponding hole by

$$
\delta=\frac{1}{4}+\frac{\mu}{4}\left(\frac{\ell}{2 \pi}\right)^{2}
$$

where the scale of the classical configuration is set by the condition $R=-\frac{\mu}{2}$ imposed on the constant scalar curvature $R$. In the case of 4 singularities at the standard locations $0, x, 1, \infty$ there are three closed geodesics $\Gamma_{s}, \Gamma_{t}, \Gamma_{u}$ separating the singular points into pairs $(x, 0 \mid 1, \infty),(x, 1 \mid 0, \infty)$ and $(x, \infty \mid 0,1)$ respectively. Since the spectrum of DOZZ theory is hyperbolic, the singularities corresponding to the saddle point weights $\delta_{i}(x)$ are geodesic holes. One may expect that these weights are related to the lengths $\ell_{i}$ of the closed geodesics $\Gamma_{i}$ in the corresponding channels:

$$
\delta_{i}(x)=\frac{1}{4}+\frac{\mu}{4}\left(\frac{\ell_{i}(x)}{2 \pi}\right)^{2}, \quad i=s, t, u .
$$

As a final remark let us point out that the conjectured formula (4.28) has strong numerical support, cf. [76]. 


\section{Conclusions}

In this work we have derived expressions for the two families of the 4-point classical blocks on the sphere. We have found, that the most non-trivial parts of the classical blocks are encoded by the Nekrasov-Shatashvili formula for the instanton part of the $\mathcal{N}=2^{*}$ U(2) twisted superpotential/eCM Yang's functional. Next, we have used one of these new expressions of the classical blocks to calculate the accessory parameter of the Fuchsian uni-

formization of the 4-punctured sphere and have found its relation to $\mathcal{W}_{\text {inst }}^{\mathcal{N}=2^{*}, \mathrm{U}(2)}$. Thirdly, we have established a relationship between the 4-point classical block on the sphere and the $N_{f}=4 \mathrm{SU}(2)$ (or $\mathrm{U}(2)$ ) twisted superpotential and further used this relationship to re-derive the Seiberg-Witten prepotential from the classical block.

There are possible extensions of the present work. Firstly, it would be interesting to apply the AGT duality and the Bethe/gauge correspondence to learn something new about the matrix models. Secondly, one can continue the studies of the triple correspondence: $2 \mathrm{dCFT} / \mathcal{N}=2$ gauge theories/quantum integrable systems going beyond $\mathrm{SU}(2)$ theories, i.e. looking at the generalization of the AGT duality to the correspondence between $2 \mathrm{~d}$ conformal Toda and $4 \mathrm{~d} \mathcal{N}=2 \mathrm{SU}(\mathrm{N})$ gauge theories [30, 31].

As a final remark let us stress that it seems to be an interesting task to study possible overlaps of our results and those in papers $[61,80]$.

\section{Acknowledgments}

The author is grateful to Paulina Suchanek, Franco Ferrari, Evgeny Ivanov and Zbigniew Jaskólski for stimulating discussions and very valuable advices. Special thanks go to Artur Pietrykowski for assistance at the initial stage of this work.

The author is also grateful to the organizers of the workshop: Branes and Bethe Ansatz in Supersymmetric Gauge Theories (Simons Center for Geometry and Physics, Stony Brook, March 2011) for the invitation and for the opportunity to present results collected in this work.

The author would like to thank the University of Szczecin and the Faculty of Mathematics and Physics of that University for the kind hospitality.

Open Access. This article is distributed under the terms of the Creative Commons Attribution Noncommercial License which permits any noncommercial use, distribution, and reproduction in any medium, provided the original author(s) and source are credited.

\section{References}

[1] A.A. Belavin, A.M. Polyakov and A.B. Zamolodchikov, Infinite conformal symmetry in two-dimensional quantum field theory, Nucl. Phys. B 241 (1984) 333 [SPIRES].

[2] T. Eguchi and H. Ooguri, Conformal and current algebras on general Riemann surface, Nucl. Phys. B 282 (1987) 308 [SPIRES].

[3] J. Teschner, An analog of a modular functor from quantized Teichmüller theory, math/0510174 [SPIRES]. 
[4] A.B. Zamolodchikov and A.B. Zamolodchikov, Structure constants and conformal bootstrap in Liouville field theory, Nucl. Phys. B 477 (1996) 577 [hep-th/9506136] [SPIRES].

[5] A.B. Zamolodchikov, Two-dimensional conformal symmetry and critical four-spin correlation functions in the Ashkin-Teller model, Sov. Phys. JETP 63 (1986) 1061.

[6] A.B. Zamolodchikov, Conformal symmetry in two-dimensional space: recursion representation of conformal block, Theor. Math. Phys. 73 (1987) 1088.

[7] L.F. Alday, D. Gaiotto and Y. Tachikawa, Liouville correlation functions from four-dimensional gauge theories, Lett. Math. Phys. 91 (2010) 167 [arXiv:0906.3219] [SPIRES].

[8] D. Gaiotto, $N=2$ dualities, arXiv:0904.2715 [SPIRES].

[9] N.A. Nekrasov, Seiberg-Witten prepotential from instanton counting, Adv. Theor. Math. Phys. 7 (2004) 831 [hep-th/0206161] [SPIRES].

[10] N. Nekrasov and A. Okounkov, Seiberg-Witten theory and random partitions, hep-th/0306238 [SPIRES].

[11] L. Hadasz, Z. Jaskólski and P. Suchanek, Modular bootstrap in Liouville field theory, Phys. Lett. B 685 (2010) 79 [arXiv:0911.4296] [SPIRES].

[12] J. Teschner, Liouville theory revisited, Class. Quant. Grav. 18 (2001) R153 [hep-th/0104158] [SPIRES].

[13] J. Teschner, A lecture on the Liouville vertex operators, Int. J. Mod. Phys. A 19S2 (2004) 436 [hep-th/0303150] [SPIRES].

[14] B. Ponsot and J. Teschner, Clebsch-Gordan and Racah-Wigner coefficients for a continuous series of representations of $U_{q}(s l(2, R))$, Commun. Math. Phys. 224 (2001) 613 [math.QA/0007097] [SPIRES].

[15] B. Ponsot and J. Teschner, Liouville bootstrap via harmonic analysis on a noncompact quantum group, hep-th/9911110 [SPIRES].

[16] A. Marshakov, A. Mironov and A. Morozov, On combinatorial expansions of conformal blocks, Theor. Math. Phys. 164 (2010) 831 [arXiv:0907.3946] [SPIRES].

[17] V. Alba and A. Morozov, Check of AGT relation for conformal blocks on sphere, Nucl. Phys. B 840 (2010) 441 [arXiv:0912.2535] [SPIRES].

[18] V.A. Fateev and A.V. Litvinov, On AGT conjecture, JHEP 02 (2010) 014 [arXiv: 0912.0504] [SPIRES].

[19] A. Mironov and A. Morozov, Proving AGT relations in the large-c limit, Phys. Lett. B 682 (2009) 118 [arXiv:0909.3531] [SPIRES].

[20] L. Hadasz, Z. Jaskólski and P. Suchanek, Proving the AGT relation for $N_{f}=0,1,2$ antifundamentals, JHEP 06 (2010) 046 [arXiv: 1004.1841] [SPIRES].

[21] G. Bonelli and A. Tanzini, Hitchin systems, $N=2$ gauge theories and $W$-gravity, Phys. Lett. B 691 (2010) 111 [arXiv: 0909.4031] [SPIRES].

[22] L.F. Alday, F. Benini and Y. Tachikawa, Liouville/Toda central charges from M5-branes, Phys. Rev. Lett. 105 (2010) 141601 [arXiv:0909.4776] [SPIRES].

[23] R. Dijkgraaf and C. Vafa, Toda theories, matrix models, topological strings and $N=2$ gauge systems, arXiv:0909.2453 [SPIRES]. 
[24] R. Poghossian, Recursion relations in CFT and N = 2 SYM theory, JHEP 12 (2009) 038 [arXiv:0909.3412] [SPIRES].

[25] A. Mironov and A. Morozov, The power of Nekrasov functions, Phys. Lett. B 680 (2009) 188 [arXiv:0908.2190] [SPIRES].

[26] A. Mironov and A. Morozov, Nekrasov functions and exact Bohr-Sommerfeld integrals, JHEP 04 (2010) 040 [arXiv:0910.5670] [SPIRES].

[27] A. Marshakov, A. Mironov and A. Morozov, Zamolodchikov asymptotic formula and instanton expansion in $N=2 S U S Y N_{f}=2 N_{c}$ QCD, JHEP 11 (2009) 048 [arXiv:0909.3338] [SPIRES].

[28] D.V. Nanopoulos and D. Xie, On crossing symmmetry and modular invariance in conformal field theory and $S$ duality in gauge theory, Phys. Rev. D 80 (2009) 105015 [arXiv: 0908.4409] [SPIRES].

[29] T.-S. Tai, Triality in SU(2) Seiberg-Witten theory and Gauss hypergeometric function, Phys. Rev. D 82 (2010) 105007 [arXiv: 1006.0471] [SPIRES].

[30] N. Wyllard, $A_{N-1}$ conformal Toda field theory correlation functions from conformal $N=2$ $\mathrm{SU}(N)$ quiver gauge theories, JHEP 11 (2009) 002 [arXiv:0907.2189] [SPIRES].

[31] A. Mironov and A. Morozov, On AGT relation in the case of $\mathrm{U}(3)$, Nucl. Phys. B 825 (2010) 1 [arXiv: 0908.2569] [SPIRES].

[32] A. Mironov, S. Mironov, A. Morozov and A. Morozov, CFT exercises for the needs of AGT, arXiv:0908.2064 [SPIRES].

[33] D. Gaiotto, Asymptotically free $N=2$ theories and irregular conformal blocks, arXiv: 0908.0307 [SPIRES].

[34] A. Marshakov, A. Mironov and A. Morozov, On non-conformal limit of the AGT relations, Phys. Lett. B 682 (2009) 125 [arXiv:0909.2052] [SPIRES].

[35] V. Alba and A. Morozov, Non-conformal limit of AGT relation from the 1-point torus conformal block, JETP Lett. 90 (2009) 708 [arXiv:0911. 0363] [SPIRES].

[36] H. Awata and Y. Yamada, Five-dimensional AGT conjecture and the deformed Virasoro algebra, JHEP 01 (2010) 125 [arXiv:0910.4431] [SPIRES].

[37] H. Awata and Y. Yamada, Five-dimensional AGT relation and the deformed beta-ensemble, Prog. Theor. Phys. 124 (2010) 227 [arXiv:1004.5122] [SPIRES].

[38] S. Yanagida, Five-dimensional SU(2) AGT conjecture and recursive formula of deformed Gaiotto state, J. Math. Phys. 51 (2010) 123506 [arXiv:1005.0216] [SPIRES].

[39] L.F. Alday, D. Gaiotto, S. Gukov, Y. Tachikawa and H. Verlinde, Loop and surface operators in $N=2$ gauge theory and Liouville modular geometry, JHEP 01 (2010) 113 [arXiv:0909.0945] [SPIRES].

[40] N. Drukker, J. Gomis, T. Okuda and J. Teschner, Gauge theory loop operators and Liouville theory, JHEP 02 (2010) 057 [arXiv:0909.1105] [SPIRES].

[41] N. Drukker, D. Gaiotto and J. Gomis, The virtue of defects in $4 D$ gauge theories and $2 D$ CFTs, arXiv: 1003.1112 [SPIRES].

[42] F. Passerini, Gauge theory Wilson loops and conformal Toda field theory, JHEP 03 (2010) 125 [arXiv: 1003.1151] [SPIRES]. 
[43] C. Kozcaz, S. Pasquetti and N. Wyllard, A and B model approaches to surface operators and Toda theories, JHEP 08 (2010) 042 [arXiv: 1004.2025] [SPIRES].

[44] H. Itoyama and T. Oota, Method of generating q-expansion coefficients for conformal block and $N=2$ Nekrasov function by beta-deformed matrix model, Nucl. Phys. B 838 (2010) 298 [arXiv: 1003.2929] [SPIRES].

[45] A. Mironov, A. Morozov and A. Morozov, Matrix model version of AGT conjecture and generalized Selberg integrals, Nucl. Phys. B 843 (2011) 534 [arXiv:1003.5752] [SPIRES].

[46] A. Morozov and S. Shakirov, The matrix model version of AGT conjecture and CIV-DV prepotential, JHEP 08 (2010) 066 [arXiv: 1004.2917] [SPIRES].

[47] P. Sulkowski, Matrix models for $\beta$-ensembles from Nekrasov partition functions, JHEP 04 (2010) 063 [arXiv:0912.5476] [SPIRES].

[48] T. Eguchi and K. Maruyoshi, Penner type matrix model and Seiberg-Witten theory, JHEP 02 (2010) 022 [arXiv:0911.4797] [SPIRES].

[49] T. Eguchi and K. Maruyoshi, Seiberg-Witten theory, matrix model and AGT relation, JHEP 07 (2010) 081 [arXiv: 1006.0828] [SPIRES].

[50] V.A. Alba, V.A. Fateev, A.V. Litvinov and G.M. Tarnopolsky, On combinatorial expansion of the conformal blocks arising from AGT conjecture, arXiv:1012.1312 [SPIRES].

[51] A. Belavin and V. Belavin, AGT conjecture and integrable structure of conformal field theory for $c=1$, Nucl. Phys. B 850 (2011) 199 [arXiv:1102.0343] [SPIRES].

[52] L. Hadasz, Z. Jaskólski and P. Suchanek, Recursive representation of the torus 1-point conformal block, JHEP 01 (2010) 063 [arXiv:0911.2353] [SPIRES].

[53] N. Seiberg and E. Witten, Monopoles, duality and chiral symmetry breaking in $N=2$ supersymmetric QCD, Nucl. Phys. B 431 (1994) 484 [hep-th/9408099] [SPIRES].

[54] G.W. Moore, N. Nekrasov and S. Shatashvili, Integrating over Higgs branches, Commun. Math. Phys. 209 (2000) 97 [hep-th/9712241] [SPIRES].

[55] A. Lossev, N. Nekrasov and S.L. Shatashvili, Testing Seiberg-Witten solution, in Cargese 1997. Strings, branes and dualities, pg. 359-372 [hep-th/9801061] [SPIRES].

[56] G.W. Moore, N. Nekrasov and S. Shatashvili, D-particle bound states and generalized instantons, Commun. Math. Phys. 209 (2000) 77 [hep-th/9803265] [SPIRES].

[57] N.A. Nekrasov and S.L. Shatashvili, Quantization of integrable systems and four dimensional gauge theories, arXiv:0908.4052 [SPIRES].

[58] N.A. Nekrasov and S.L. Shatashvili, Supersymmetric vacua and Bethe ansatz, in Cargese 2008. Theory and particle physics: the LHC perspective and beyond, Nucl. Phys. Proc. Suppl. 192-193 (2009) 91 [arXiv:0901.4744] [SPIRES].

[59] N.A. Nekrasov and S.L. Shatashvili, Quantum integrability and supersymmetric vacua, Prog. Theor. Phys. Suppl. 177 (2009) 105 [arXiv:0901.4748] [SPIRES].

[60] C.-N. Yang and C.P. Yang, Thermodynamics of a one-dimensional system of bosons with repulsive delta-function interaction, J. Math. Phys. 10 (1969) 1115 [SPIRES].

[61] J. Teschner, Quantization of the Hitchin moduli spaces, Liouville theory and the geometric Langlands correspondence I, arXiv:1005.2846 [SPIRES]. 
[62] A.B. Zamolodchikov, Conformal symmetry in two-dimensions: an explicit recurrence formula for the conformal partial wave amplitude, Commun. Math. Phys. 96 (1984) 419 [SPIRES].

[63] A.B. Zamolodchikov, A.B. Zamolodchikov and I.M. Khalatnikov, Conformal field theory and critical phenomena in two-dimensional systems, Sov. Sci. Rev. A Phys. 10 (1989) 269 [SPIRES].

[64] T.-S. Tai, Uniformization, Calogero-Moser/Heun duality and Sutherland/bubbling pants, JHEP 10 (2010) 107 [arXiv: 1008.4332] [SPIRES].

[65] E. Picard, De l'équation $\Delta u=k \mathrm{e}^{u}$ sur une surface de Riemann fermée, J. Math. Pure Appl. 9 (1893) 273.

[66] E. Picard, De l'intégration de l'équation $\Delta u=\mathrm{e}^{u}$ sur une surface de Riemann fermée, Crelle's J. 130 (1905) 243.

[67] M. Troyanov, Prescribing curvature on compact surfaces with conical singularities, Trans. Am. Math. Soc. 324 (1991) 793.

[68] L.V. Ahlfors, Complex analysis, third edition, McGrawHill, Kogakusha Tokyo Japan (1979).

[69] J.A. Hempel, On the uniformization of the n-punctured sphere, Bull. London Math. Soc. 20 (1988) 97.

[70] L. Takhtajan and P. Zograf, Hyperbolic 2-spheres with conical singularities, accessory parameters and Kähler metrics on $\mathcal{M}_{0, n}$, Trans. Am. Math. Soc. 355 (2003) 1857 [math.CV/0112170] [SPIRES].

[71] L.A. Takhtajan, Topics in quantum geometry of Riemann surfaces: two-dimensional quantum gravity, in Como Quantum Groups 1994, pg. 541-580 [hep-th/9409088] [SPIRES].

[72] P.G. Zograf and L.A. Takhtajan, On Liouville equation, accessory parameters and the geometry of Teichmüller space for Riemann surface of genus 0 , Math. USSR Sbornik 60 (1988) 143.

[73] L. Cantini, P. Menotti and D. Seminara, Proof of Polyakov conjecture for general elliptic singularities, Phys. Lett. B 517 (2001) 203 [hep-th/0105081] [SPIRES].

[74] L. Hadasz and Z. Jaskólski, Polyakov conjecture for hyperbolic singularities, Phys. Lett. B 574 (2003) 129 [hep-th/0308131] [SPIRES].

[75] L. Hadasz and Z. Jaskólski, Classical Liouville action on the sphere with three hyperbolic singularities, Nucl. Phys. B 694 (2004) 493 [hep-th/0309267] [SPIRES].

[76] L. Hadasz, Z. Jaskólski and M. Piạtek, Classical geometry from the quantum Liouville theory, Nucl. Phys. B 724 (2005) 529 [hep-th/0504204] [SPIRES].

[77] M. Piątek, Analytic properties of conformal blocks, Ph.D. thesis (2006).

[78] L. Hadasz and Z. Jaskólski, Liouville theory and uniformization of four-punctured sphere, J. Math. Phys. 47 (2006) 082304 [hep-th/0604187] [SPIRES].

[79] N. Seiberg, Notes on quantum Liouville theory and quantum gravity, in Common trends in mathematics and quantum field theory, Proceedings of the 1990 Yukawa International Seminar, Prog. Theor. Phys. Suppl. 102 (1990) 319 [SPIRES].

[80] N. Nekrasov, A. Rosly and S. Shatashvili, Darboux coordinates, Yang-Yang functional and gauge theory, arXiv:1103.3919 [SPIRES]. 